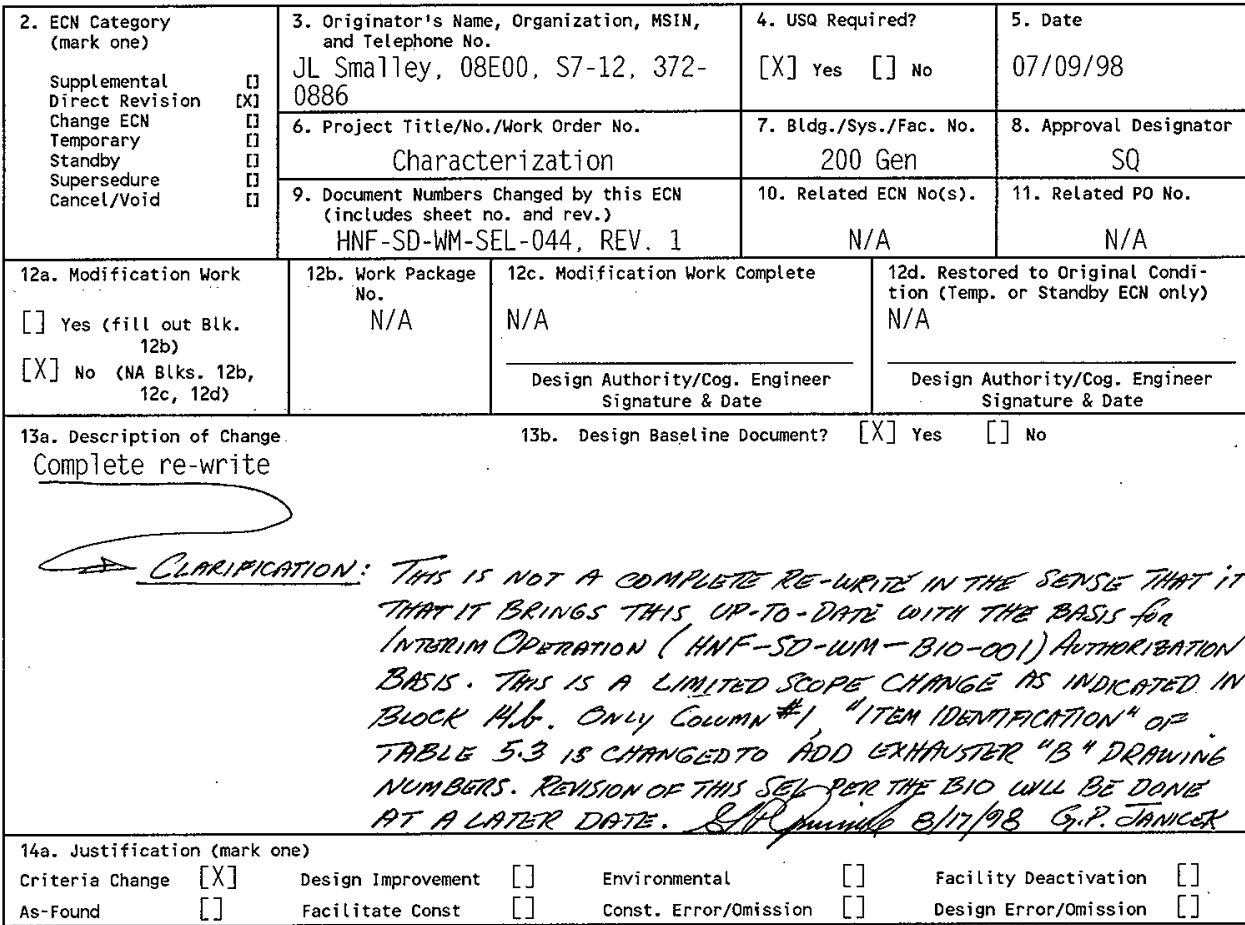

14b. Justification Details

THIS CHANGE IS BEING MADE TO INCLUDE THE EXHAUSTER B DRAWING NUMBERS FOR EQUIPMENT IDENTIFICATION OF THE SAFETY CLASS COMPONENTS ON EXHAUSTER $B$.

SEE USQ TF-97-0939 REVISFON $6.7 k+$

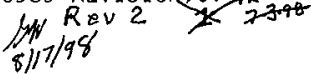

15. Distribution (include name, MSIN, and no. of copies)

Jl Smalley

CE Hanson

JD Criddle

RM Boger

KV Scott

JA Ranschau

GW Wi]son
S7. -12

$57-12$

$57-12$

$57-12$

S7 -12

S7-07

S7 -12
ML MCEIroy

us Schofield

GP Janicek.

EJ Waldo

TD Jareckj

RR Bafus

JR KRiskovich
S7 -07

S7 -12

S7-12

S7-12

S7-03

S7-12

R1- 56

A-7900-013-2 (05/96) GEF095 


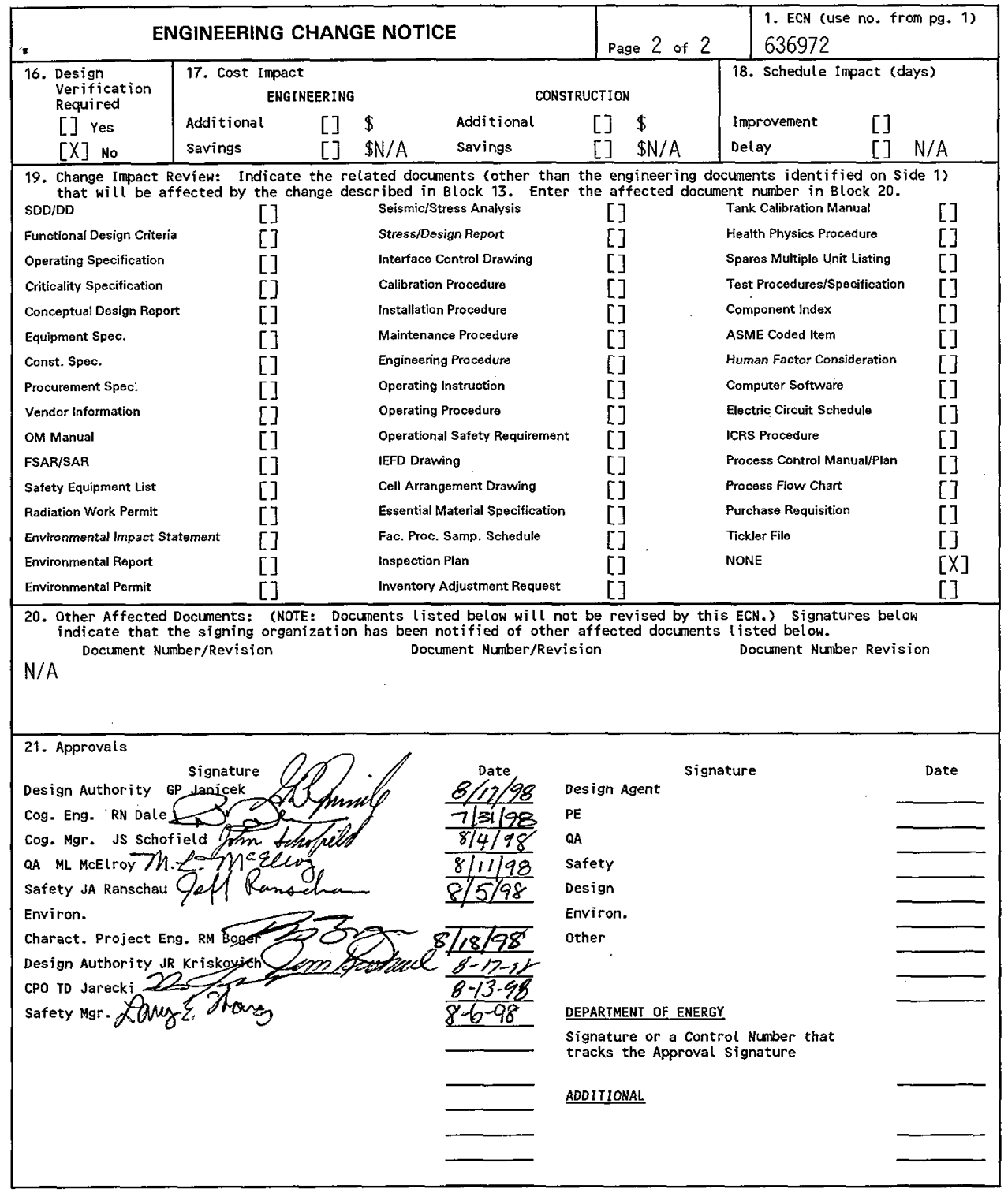




\section{A Safety Equipment List for Rotary Mode Core Sampling Systems Operation in Single Shell Flammable Gas Tanks}

JL Sma1ley

SGN Eurisys Services Corporation, Richland, WA 99352

U.S. Department of Energy Contract DE-AC06-96RL13200

EDT/ECN: 636970

Org Code: $08 \mathrm{E} 00$

UC: 2070

B\&R Code: EW3120074

Charge Code: N4H2D

Total Pages: 49

Key Words: Safety Equipment List, SEL, Rotary Mode Core Sampling System, RMCSS, single she11, flammable gas tanks.

Abstract: This document identifies al1 interim safety equipment to be used for rotary mode core sampling of single-shell flammable gas tanks utilizing Rotary Mode Core Sampling Systems (RMCSS)

TRADEMARK DISCLAIMER. Reference herein to any specific comercial product, process, or service by trade name, trademark, manufacturer, or otherwise, does not necessarily constitute or inply its endorsement, recommendation, or favoring by the United States Government or any agency thereof or its contractors or subcontractors.

Printed in the United States of America. To obtain copies of this document, contact: Document Control Services, P.0. BoX 950, Mailstop H6-08, Richland WA 99352, Phone (509) 372-2420;

Fax (509) 376-4989.
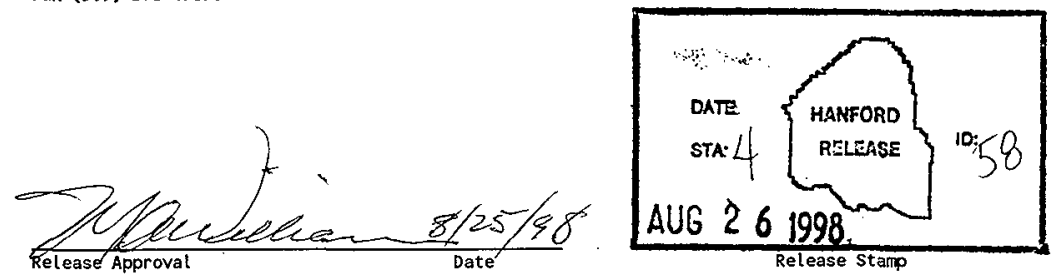

Approved for Public Release 
(2) Title

A Safety Equipment List for Rotary Mode Core Sampling Systems Operation in Single Shell Flammable Gas Tanks

CHANGE CONTROL RECORD

\begin{tabular}{|l|l|} 
(3) Revision & (4) Description of Change - Replace, Add, and Delete Pages \\
\hline 0 & (7) EDT 622107 \\
\hline 1 & Complete re-write (ECN 636970) \\
\hline
\end{tabular}

2 RS Added Exhauster $B$ drawing numbers to the equipment identification column of section 5.3 Safety Equipment List for the RMCS System Exhauster. Also added a note to section 3.0 explaining why the exhauster equipment has more than one identification number. (direct revision ECN 636972)
Authorized for Release

(5) Cog. Engr. (6) Cog. Mgr. Date

R.N. Dale J.S. Schofield

R.N. Dale J.S. Schofield 12/11/97 $12 / 11 / 97$

R.N. Dale J.S. Schofield

RNDen $7 / 31198$

$8 / 3 / 98$

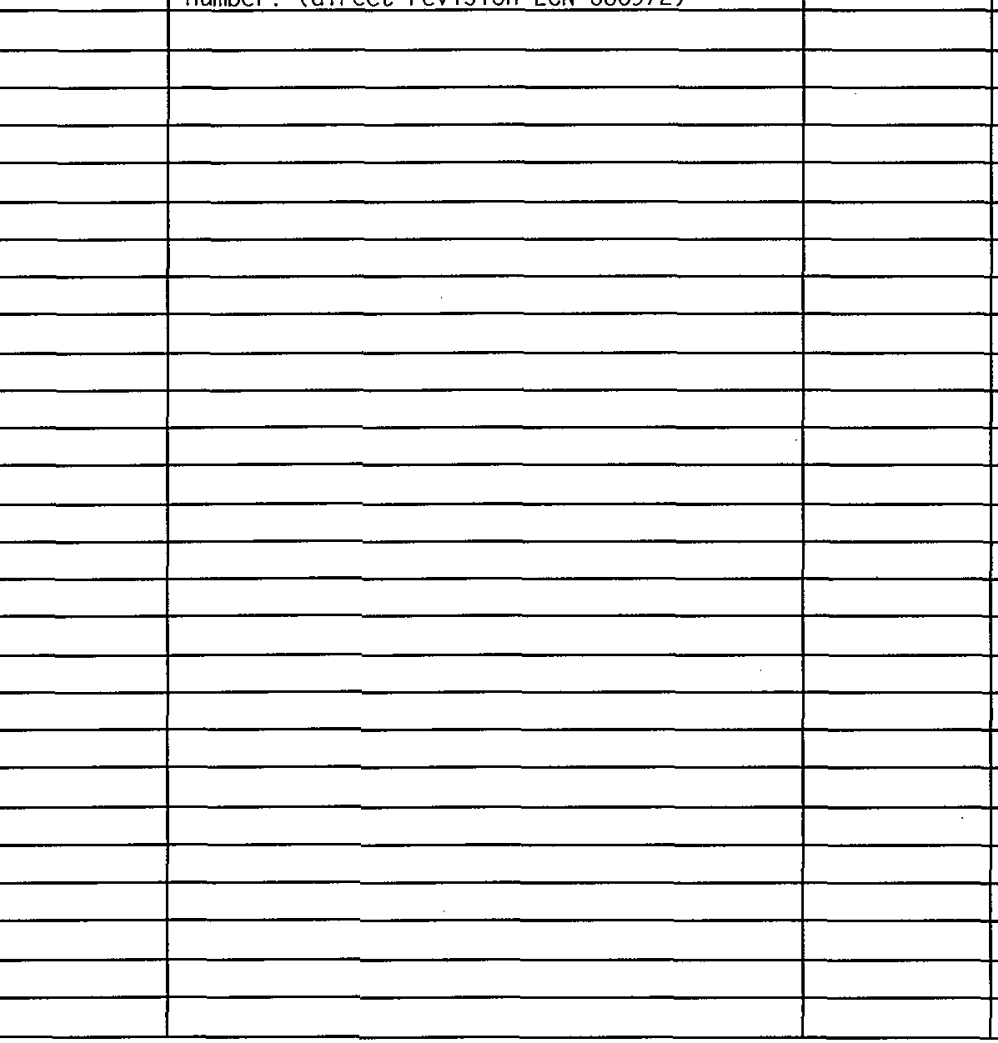


A SAFETY EQUIPMENT LIST FOR ROTARY MODE CORE

\author{
SAMPLING SYSTEMS OPERATION IN \\ SINGLE-SHELL \\ FLAMMABLE GAS TANKS
}

HNF-SD-WM-SEL-044

REVISION 2

J. L. Smalley

COGEMA ENGINEERING CORPORATION 
HNF-SD-WM-SEL-044, Rev. 2

Page 2 of 47

July 1998

Table of Contents

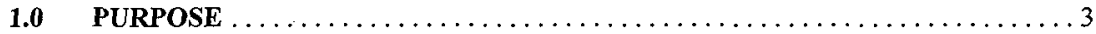

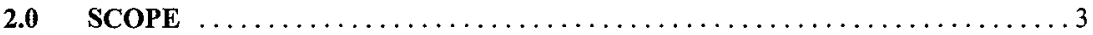

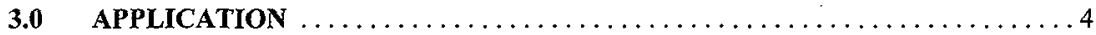

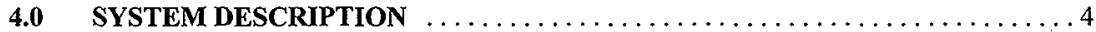

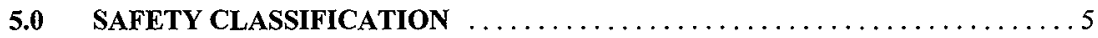

5.1 SAFETY EQUIPMENT LIST FOR THE RMCS SYSTEM CORE SAMPLE

TRUCK ..................................

5.1.1 SAFETY EQUIPMENT LIST FOR THE SHIELDED RECEIVER SYSTEM OF THE RMCS CORE SAMPLE TRUCK .......... 12

5.1.2 SAFETY EQUIPMENT LIST FOR THE GRAPPLE HOIST ASSEMBLY OF THE RMCS CORE SAMPLE TRUCK . . . . . . 17

5.1.3 SAFETY EQUIPMENT LIST FOR THE PURGE GAS INSTRUMENTATION SYSTEM OF THE CORE SAMPLE TRUCK

5.1.4 SAFETY EQUIPMENT LIST FOR THE DOWNWARD FORCE INSTRUMENTATION SYSTEM OF THE CORE SAMPLE TRUCK ................................... 26

5.1.5 SAFETY EQUIPMENT LIST FOR THE RPM INSTRUMENTATION SYSTEM OF THE CORE SAMPLE TRUCK . ............28

5.1.6 SAFETY EQUIPMENT LIST FOR THE PENETRATION RATE INSTRUMENTATION OF THE CORE SAMPLE TRUCK . . . . . . . 29

5.1.7 SAFETY EQUIPMENT LIST FOR THE HYDRAULIC CONTROL INSTRUMENTATION OF THE CORE SAMPLE TRUCK ........ 30

5.1.8 SAFETY EQUIPMENT LIST FOR THE SHUTDOWN INTERLOCK OF THE CORE SAMPLE TRUCK ..................... 33

5.1.9 SAFETY EQUIPMENT LIST FOR THE DRILL STRING COMPONENTS OF THE CORE SAMPLE TRUCK .......... 35

5.2 SAFETY EQUIPMENT LIST FOR THE RMCS SYSTEM RISER EQUIPMENT

5.3 SAFETY EQUIPMENT LIST FOR THE RMCS SYSTEM EXHAUSTER . . . 38

5.4 SAFETY EQUIPMENT LIST FOR THE RMCS SYSTEM UNIVERSAL

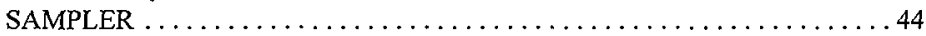


HNF-SD-WM-SEL-044, Rev. 2

Page 3 of 47

\section{A Safety Equipment List for Rotary Mode Core Sampling Systems Operation in Single-Shell Flammable Gas Tanks}

\subsection{PURPOSE}

This document identifies all interim safety equipment to be used for rotary mode core sampling of single-shell flammable gas tanks utilizing Rotary Mode Core Sampling systems (RMCS).

\section{$2.0 \quad$ SCOPE}

This document provides the safety equipment for RMCS trucks HO-68K-4600, HO-68K-4647, trucks three and four respectively, and associated equipment. It is not intended to replace or supersede WHC-SD-WM-SEL-023, (Kelly 1991), or WHC-SD-WM-SEL-032, (Corbett 1994), which classifies HO-68K-4344 and HO-68K-4345 respectively. The term "safety equipment" refers to safety class (SC) and safety significant (SS) equipment, where equipment refers to structures, systems and components (SSC's).

The identification of safety equipment in this document is based on the credited design safety features and analysis contained in the Authorization Basis (AB) for rotary mode core sampling operations in single-shell flammable gas tanks. This is an interim safety classification since the $\mathrm{AB}$ is interim. This document will be updated to reflect the final RMCS equipment safety classification designations upon completion of a final $A B$ which will be implemented with the release of the Final Safety Analysis Report (FSAR).

The current interim $\mathrm{AB}$ for rotary mode core sampling in single-shell flammable gas tanks utilizing RMCS systems three and four (RMCS trucks HO-68K-4600 and HO-68K-4647) is the Basis for Interim Operation (BIO) (LMHC 1997b), Wagoner 1997b, the RMCS initial Safety Evaluation Report (DOE-RL 1996), Wagoner 1996, LMHC 1997e, WHC-SD-WM-SAD-035( LMHC 1997d), and the Technical Safety Requirements (TSRs) (LMHC 1997f) with clarification from Wagoner 1997c. Wagoner 1996, was the first approval document for performing RMCS in flammable gas tanks. Wagoner 1996, referenced WHC-SD-WM-SAD-035, the initial Safety Evaluation Report (SER,) and itself as being part of the authorization basis. The initial SER states that WHC-SD-WM-SAD-035 (LANL 1996), WHC-SD-WM-OSR-005 (LMHC 1996), and the SER, itself, provide the Authorization Basis for RMCS. In July 1997, DOE issued Wagoner 1997b. This second SER approved deletion of a number of flammable gas controls for RMCS from LANL 1996 and LMHC 1996, and approved addition of the Standing Order flammable gas controls in LMHC 1997a. The Standing Order flammable gas controls and some of the OSR controls were replaced by the BIO/TSR controls on September 29, 1997. 
The listing shown in section 5.0 is a summary of the safety classification for all safety equipment for these systems. The actual safety equipment list (SEL), including safety classification justification, is identified in sections 5.1-5.4. All equipment associated with this system not listed in this document is considered non-safety class equipment and shall be designated as General Service (GS).

\subsection{APPLICATION}

The safety classification designation will be used as the basis for confirming the appropriate Quality, Safety, and Environmental Assurance, and Quality Control is used for design work, procurement, installation/modification, maintenance, and operation of equipment. Each listing contains: identification of the equipment, functional description of the equipment, equipment safety function, failure mechanisms, design attributes, failure effect on SSC safety function, safety classification justification, and designated safety classification. Note, section 5.3 (Safety Equipment List for the RMCS System Exhauster) contains up to three identification numbers for each item in the list. One identification number is for Exhauster B, the other is for Exhauster C and the remaining number is a reference number. The reference numbers were put in the list to reflect previous designations for the equipment that were used prior to the Exhauster drawings being updated. These numbers were used on the original Commercial Grade Item dedication forms and are left here for traceability only.

\subsection{SYSTEM DESCRIPTION}

The RMCS systems three and four consist of the sampling truck, the portable exhauster, the nitrogen supply system, the portable generator, the $\mathrm{x}$-ray machine, the power distribution trailer, support trucks, support trailer, universal samplers, and associated ancillary equipment.

The RMCS systems are specifically designed to rotary sample in single-shell flammable gas tanks. The waste is collected in a universal sampler, which is lowered down through the drill string and seals in the drill bit section. While the drill bit penetrates and the sampler descends, a piston in the sampler is held in place, allowing the core sample to be drawn into the sampler. Nitrogen purge gas cools the bit and clears away the cuttings during drilling. This gas is exhausted and filtered by a tank ventilation system to avoid particulate emissions from the tank. A dedicated portable exhauster can be connected to a separate riser for this purpose on tanks that are not actively ventilated. A system is in place to provide nitrogen hydrostatic head gas to the drill string to prevent waste flooding during sampler change out. 
When cutting through the waste material, friction and heat can be generated by the drill bit causing waste heating and possible ignition of the waste. Therefore, the drill bit temperature must be limited to eliminate the potential reactivity of the tank contents during sampling operations. The drill bit temperature is controlled by enveloping the operating parameters which affect drill bit temperature. Identification of the safety envelope and justification for the alarm set points and alarm delays are contained in the $A B$.

For further system description details refer to (WHC 1995) and (Robinson 1995).

\subsection{SAFETY CLASSIFICATION}

The following listing is a summary of the safety equipment associated with RMCS operations in single-shell flammable gas tanks. For specific information about the individual components listed refer to the specified section of this document. The design attributes for the safety SSC's are listed in sections 5.1-5.4. These attributes are critical to the SSC safety function, as analyzed in the referenced safety documentation. In some cases the design attributes listed are the SSC "Critical Characteristics," as defined in HNF-PRO-447 (HNF 1997a).

\section{SAFETY EQUIPMENT LIST SUMMARY}

Rotary Mode Core Sampling Truck

Shielded Receiver Assembly

SC/See section 5.1

Grapple Hoist

SC/See section 5.1

Purge Gas Instrumentation SC/See section 5.1

Downward Force Instrumentation $\mathrm{SC} /$ See section 5.1

RPM Instrumentation

SC/See section $\$ .1$

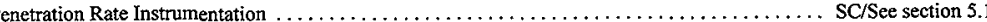

Hydraulic Controls Instrumentation $\ldots \ldots \ldots \ldots \ldots \ldots \ldots \ldots \ldots \ldots \ldots \ldots \ldots \ldots \ldots \ldots \ldots \ldots \ldots \ldots \ldots \ldots$ SC/See setion 5.1

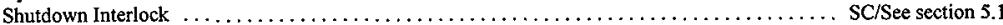

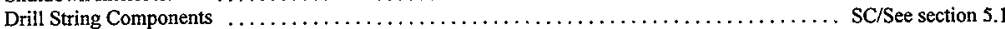

Shielded Receiver Assembly $\ldots \ldots \ldots \ldots \ldots \ldots \ldots \ldots \ldots \ldots \ldots \ldots \ldots \ldots \ldots \ldots \ldots \ldots \ldots \ldots \ldots \ldots \ldots \ldots$ SC See section 5.1 .1

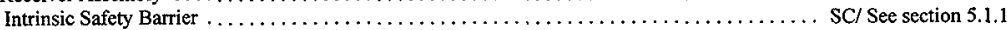

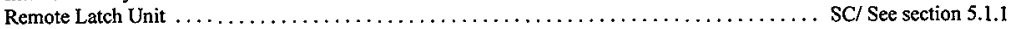

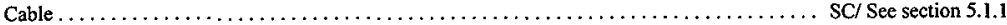

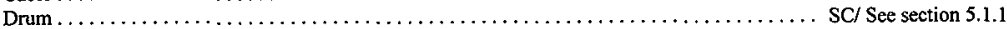

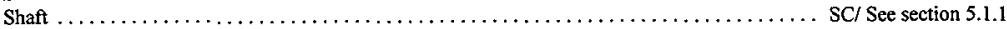

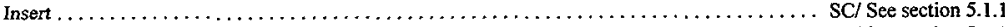

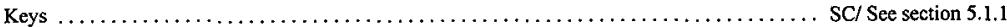

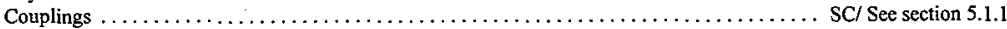

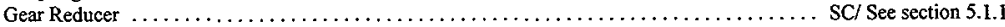

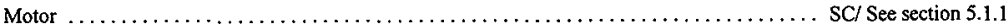

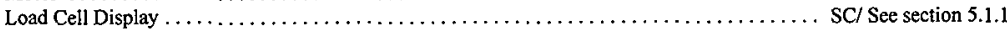

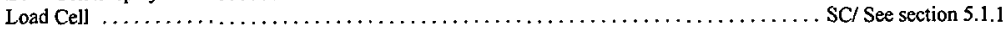


Intrinsic Safety Barrier

$\mathrm{SC} /$ See section 5.1 .2

Sample Actuator (grapple)

SC/ See section 5.1.2

Wire Rope

$\mathrm{SC} /$ See section 5.1.2

Drum

$\mathrm{SC} /$ See section 5.1.2

Shaft

SC/ See section 5.1.2

Roll Pin .

$\mathrm{SC} /$ See section 5.1.2

Ke

$\mathrm{SC} /$ See section 5.1 .2

Coupling .

SC/ See section 5.1.2

Gear Reducer

$\mathrm{SC} /$ See section 5.1 .2

Motor

SC/ See section 5.1.2

Motor Controller

$\mathrm{SC} /$ See section 5.1.2

Load Cell Display

$\mathrm{SC} /$ See section 5.1.2

Load Cell

SC/ See section 5.1.2

Purge Gas Instrumentation

$\mathrm{SC} /$ See section 5.1 .3

Purge Gas Flow Meter

$\mathrm{SC} /$ See section 5.1.3

Purge Gas Flow Turbine

$\mathrm{SC} /$ See section 5.1 .3

Purge Gas Flow Pressure Transducer

$\mathrm{SC} /$ See section 5.1 .3

Purge Gas Flow Temperature Transmitter

$\mathrm{SC} /$ See section 5.1 .3

Riser Sleeve Purge Differential Pressure Switch

$\mathrm{SC/}$ See section 5.1.3

Purge Gas Temperature Display

SS/ See section 5.1.3

Purge Gas Temperature Thermocouple

$\mathrm{SS} /$ See section 5.1 .3

Pressure Switch

$\mathrm{SC} /$ See section 5.1 .3

Downward Force Instrumentation

$\mathrm{SC} /$ See section 5.1 .4

Downward Force Pressure Transducers

SC/ See section 5.1.4

Downward Force Adder/Subtractor

$\mathrm{SC} /$ See section 5.1.4

Downward Force Digital Display .

$\mathrm{SC} /$ See section 5.1.4

RPM Instrumentation

$\mathrm{SC} / \mathrm{See}$ section 5.1 .5

Drill Rotation Sensor

$\mathrm{SC} /$ See section 5.1 .5

RPM Digital Display Unit

$\mathrm{SC} /$ See section 5.1 .5

Penetration Rate Instrumentation

SC/ See section 5.1.6

Drill Bit Penetration Digital Display Unit

$\$ \mathrm{C} /$ See section 5.1.6

Magneto Tachometer Generator

$\mathrm{SC} /$ See section 5.1 .6

Hydraulic Control Instrumentation

SC/ See section 5.1.7

Lower Ram Pressure Display

SC/ See section 5.1 .7

HBD/Walkdown Function Pressure Transducer

SC/ See section 5.1.7

HBD Hydraulic Solenoid Valve

SC/ See section 5.1.7

Walkdown Function Solenoid Valve .

SC/ See section 5.1.7

HBD/Walkdown Function Comparator

SC/ See section 5.1.7

Shutdown Interlock

SC/ See section 5.1.8

PLC

SC/ See section 5.1 .8

Shutdown Interlock (K5 Relay) .

SC/ See section 5.I.8

Exhauster/Interlock Shutdown Overrid

$\mathrm{SS} /$ See section 5.1 .8

Pressure Switch for Actively Ventilated Tanks $\mathrm{SC} /$ See section 5.1 .8 
Fan $\mathrm{SC} /$ See section 5.3

Pressure Switch ISB . SC/See section 5.3

Differential Pressure Switch SC/See section 5.3

HEPA filter unit SS/See section 5.3

Inlet Duct $\mathrm{SC} / \mathrm{See}$ section 5.3

Relative Humidity Transmitter SC/See section 5.3

Tank Pressure Switch $\mathrm{SC} / \mathrm{See}$ section 5.3

Masstron Stack Flow Transmitter $\mathrm{SC} /$ See section 5.3

Masstron Sample Flow Transmitter SC/See section 5.3

Veltron Vacuum Transmitter SC/See section 5.3

Vacuum Pump Pressure Switches SC/See section 5.3

Vacuum Pumps SC/See section 5.3

Truck Shutdown Relay SS/See section 5.3

Variable Frequency Drive SS/See section 5.3

Exhauster Low Flow Relay SS/See section 5.3

Exhauster Flow Controller SS/See section 5.3

Beta CAM In-Line Remote Detector Head SC/See section 5.3 


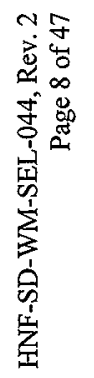

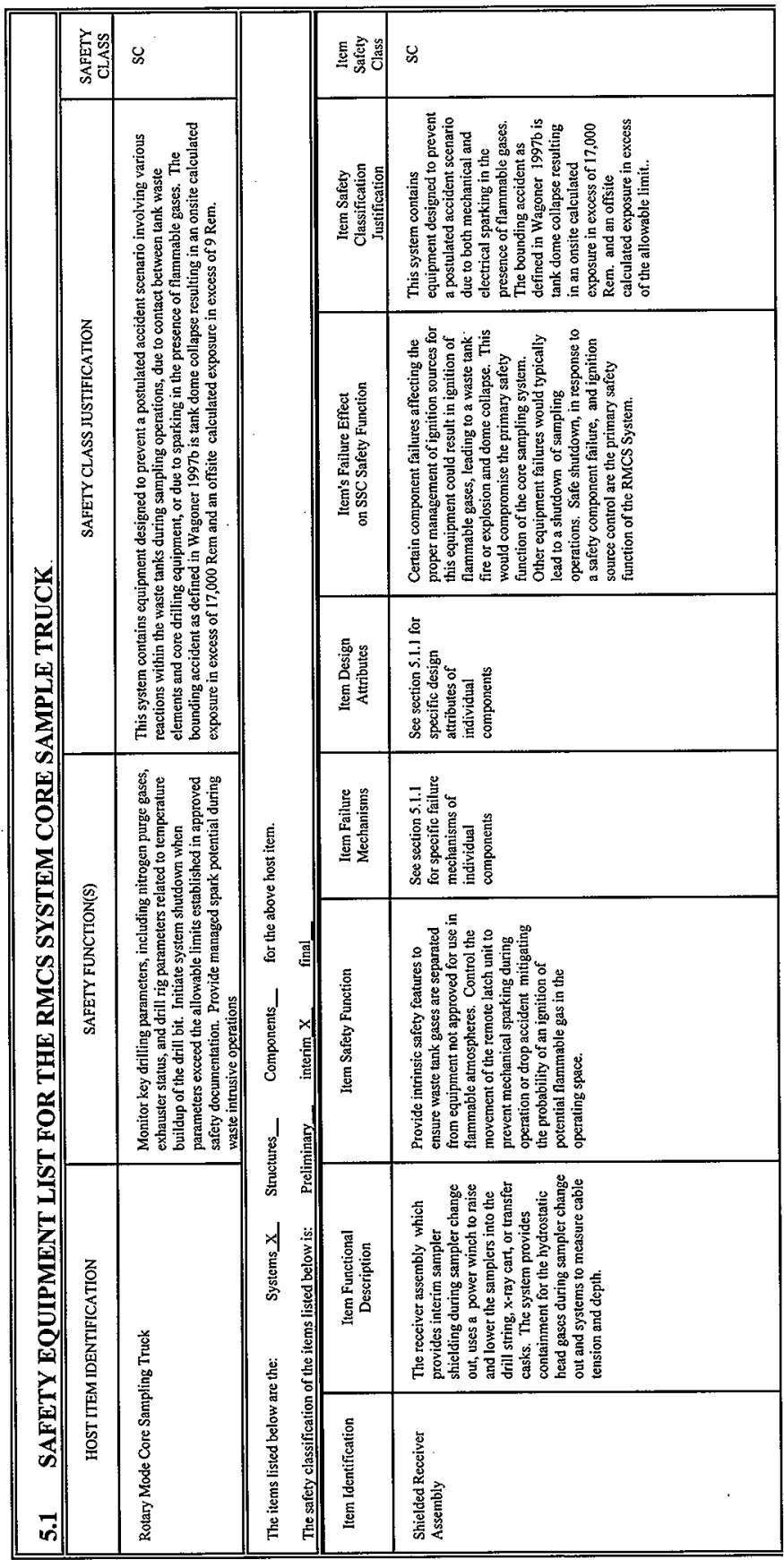




\begin{tabular}{|c|c|c|c|c|}
\hline & 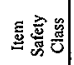 & U্口 & 迎 & Un \\
\hline & 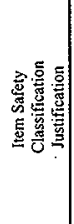 & 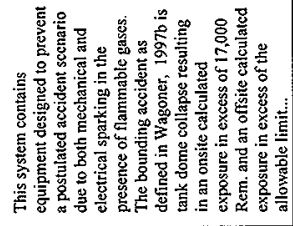 & 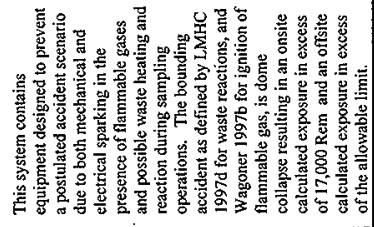 & 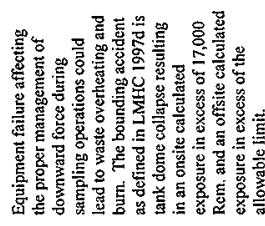 \\
\hline ક્犬્犬 & 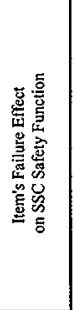 & 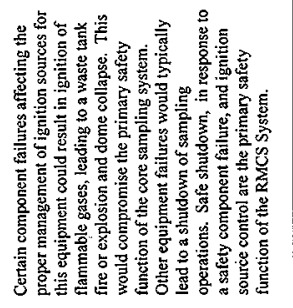 & 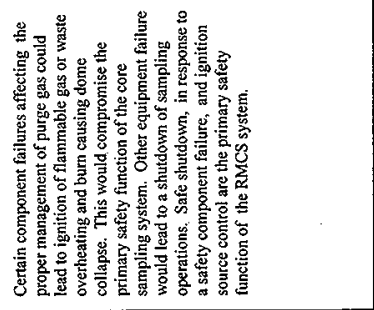 & 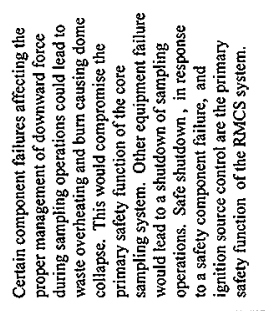 \\
\hline 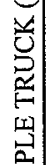 & 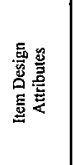 & 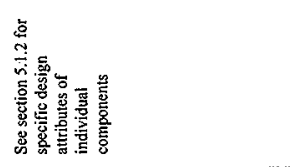 & 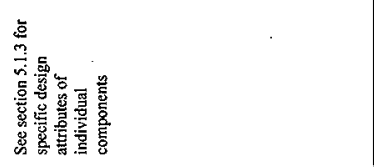 & 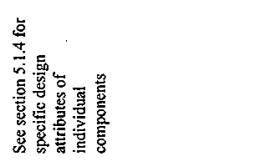 \\
\hline 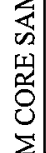 & 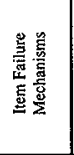 & 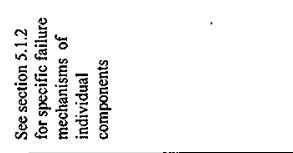 & 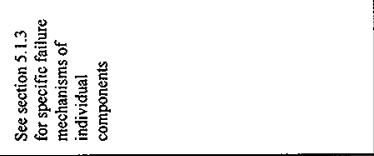 & 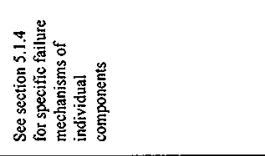 \\
\hline 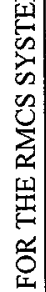 & 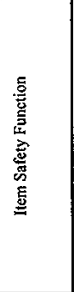 & 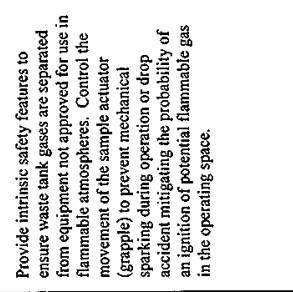 & 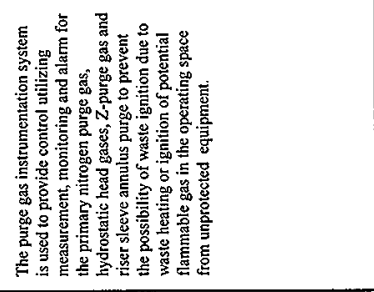 & 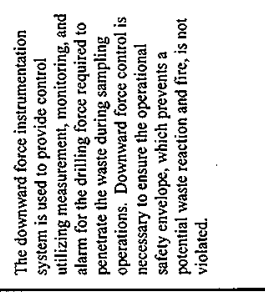 \\
\hline 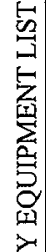 & 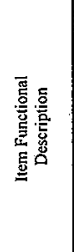 & 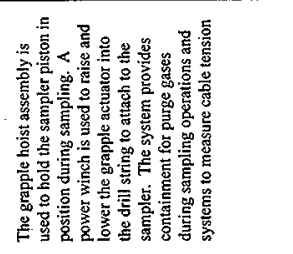 & 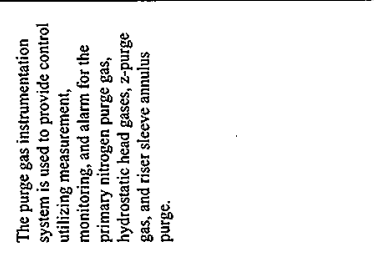 & 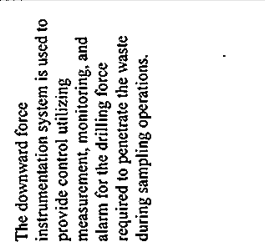 \\
\hline 紊 & 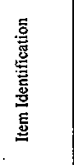 & 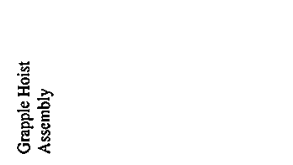 & 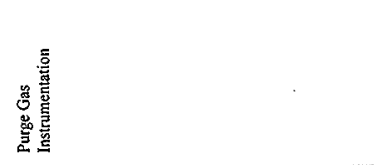 & 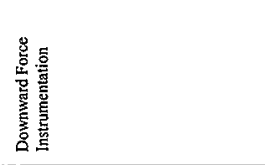 \\
\hline
\end{tabular}




\begin{tabular}{|c|c|c|c|c|}
\hline & 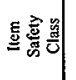 & U & 佂 & $\ddot{m}$ \\
\hline & 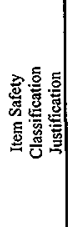 & 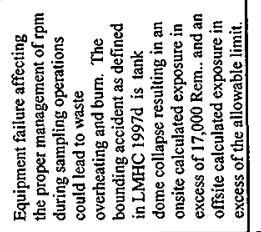 & 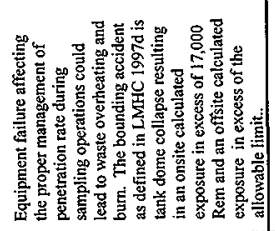 & 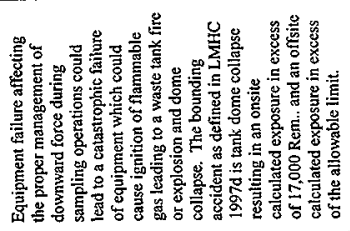 \\
\hline$\underbrace{3}_{0}$ & 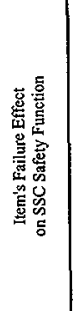 & 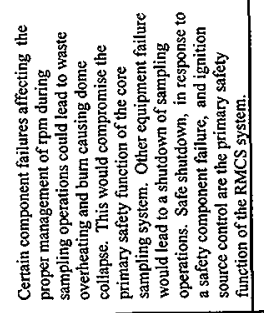 & 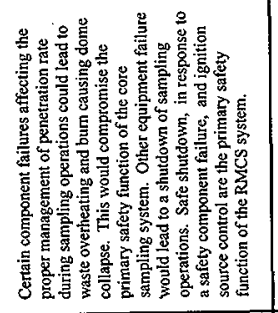 & 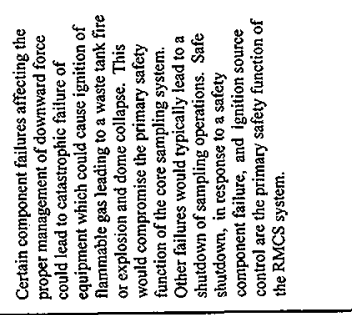 \\
\hline 量 & 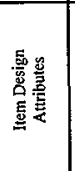 & 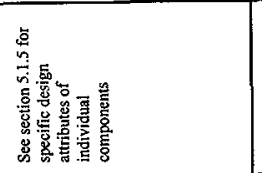 & 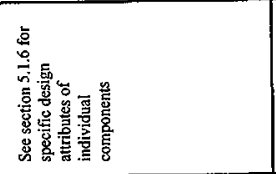 & 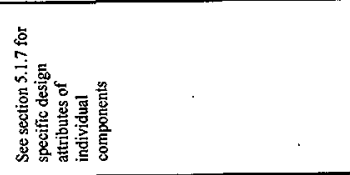 \\
\hline 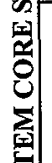 & 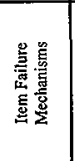 & 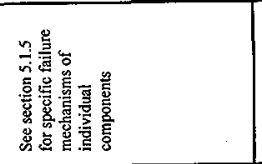 & 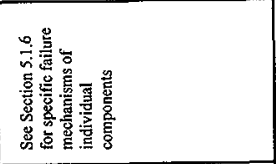 & 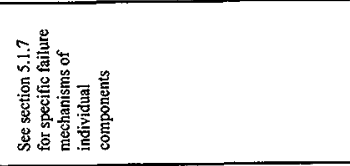 \\
\hline 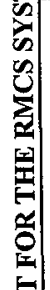 & 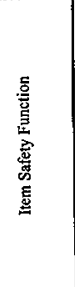 & 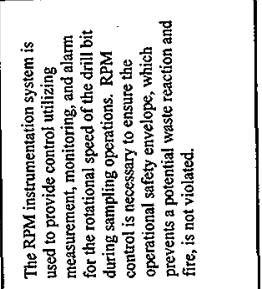 & 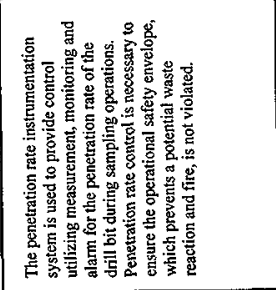 & 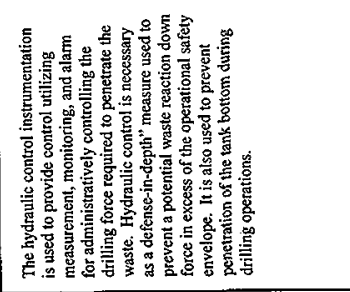 \\
\hline |a & 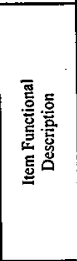 & 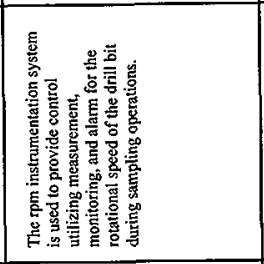 & 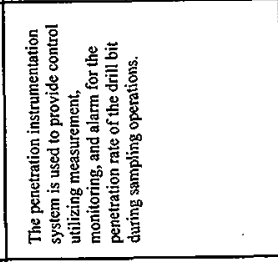 & 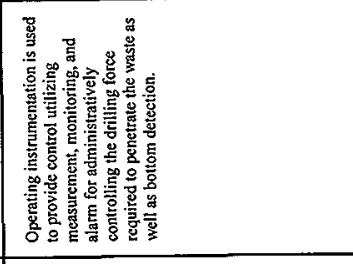 \\
\hline 帘 & 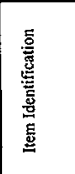 & 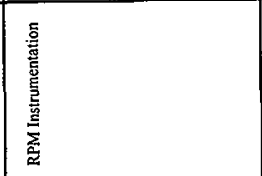 & 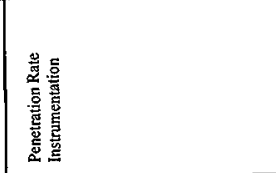 & 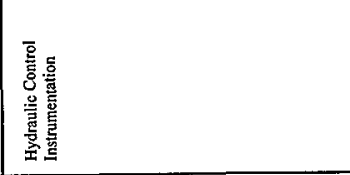 \\
\hline
\end{tabular}




\begin{tabular}{|c|c|c|c|}
\hline & 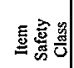 & Ü & U \\
\hline & 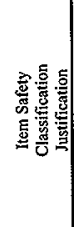 & 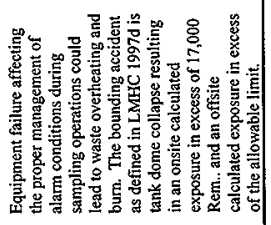 & 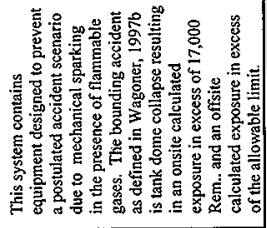 \\
\hline 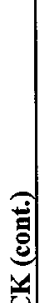 & 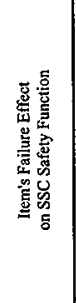 & 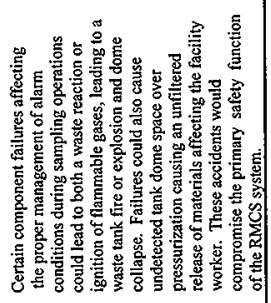 & 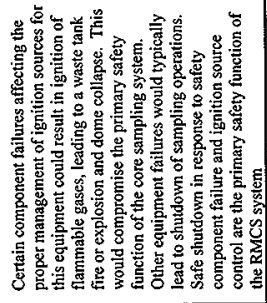 \\
\hline 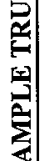 & 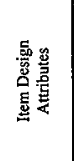 & 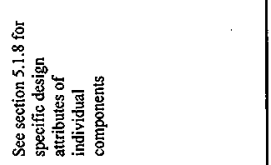 & 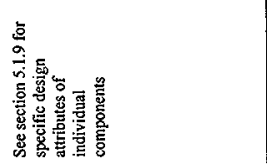 \\
\hline $\begin{array}{l}\text { 图 } \\
0 \\
0 \\
0 \\
\end{array}$ & 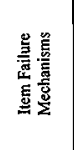 & 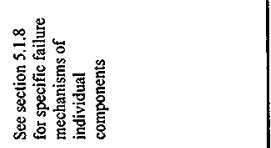 & 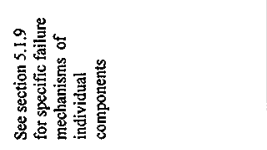 \\
\hline 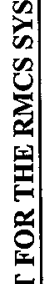 & 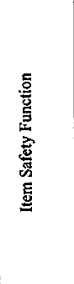 & 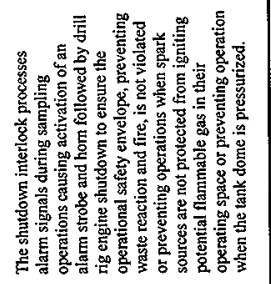 & 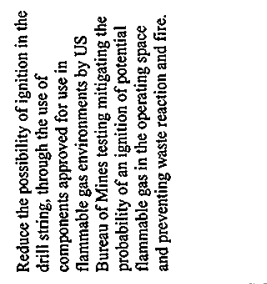 \\
\hline 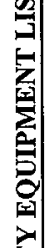 & 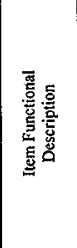 & 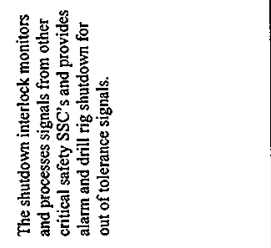 & 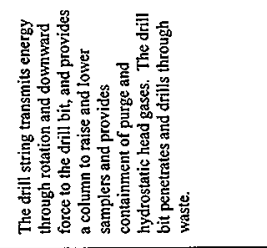 \\
\hline$\frac{1}{a}$ & 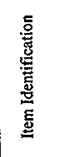 & 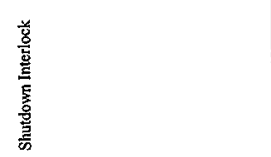 & 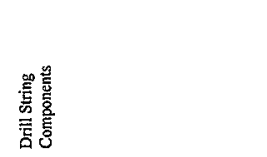 \\
\hline
\end{tabular}




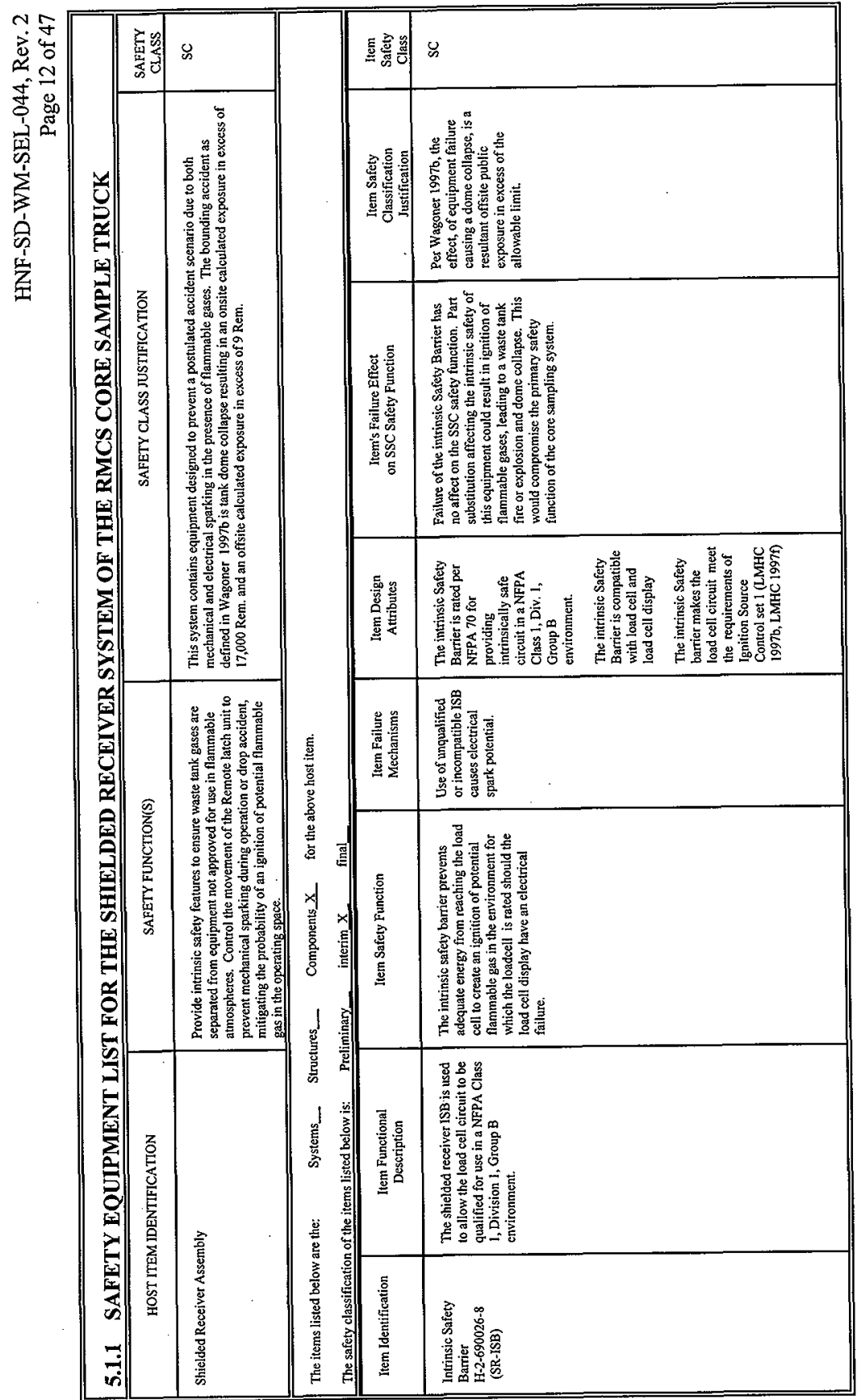




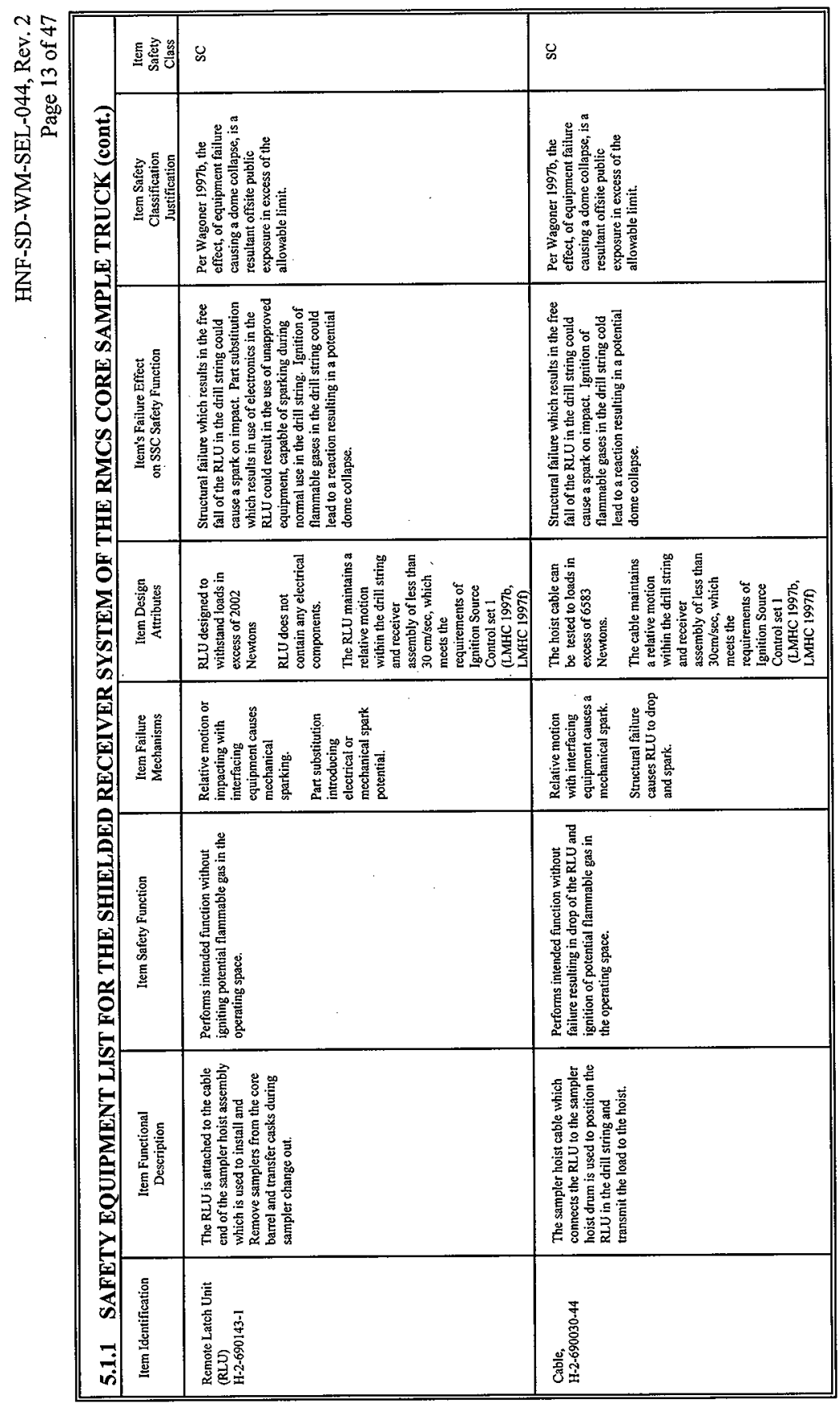




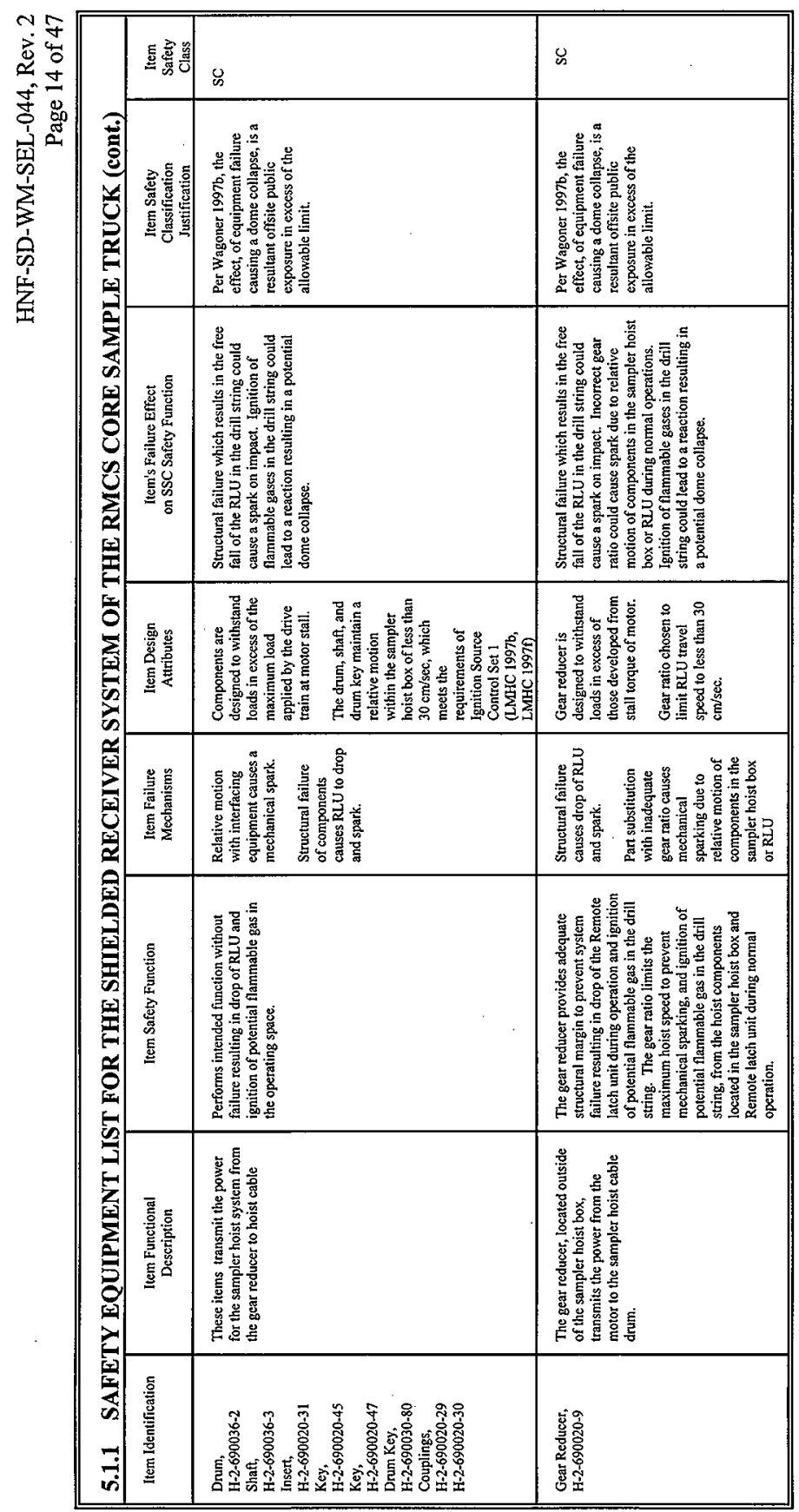




\begin{tabular}{|c|c|c|c|c|c|}
\hline & 镸总哭 & o & $\mathscr{E}$ & & \\
\hline 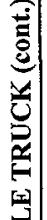 & 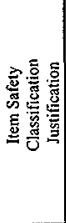 & 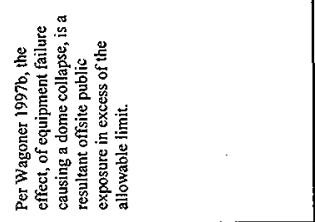 & 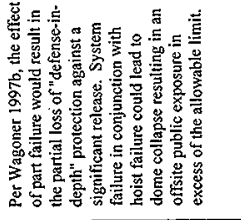 & & \\
\hline 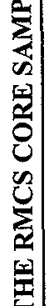 & 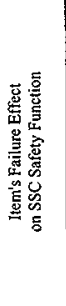 & 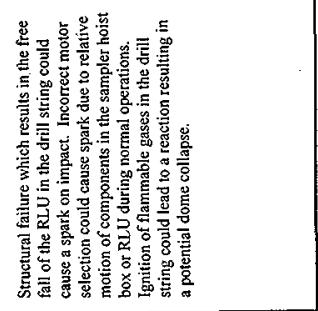 & 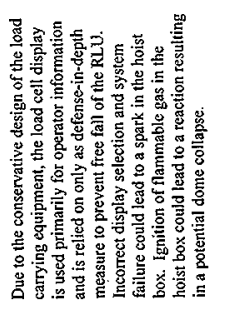 & & \\
\hline$\sum_{\substack{\infty \\
0}}^{\substack{6 \\
\infty}}$ & 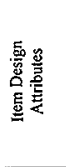 & 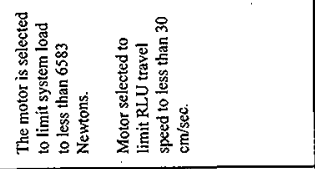 & 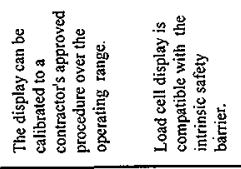 & & \\
\hline 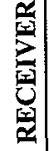 & 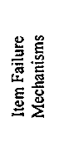 & 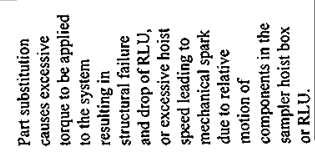 & 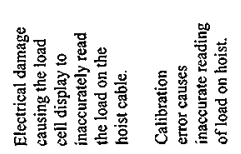 & 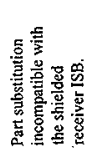 & 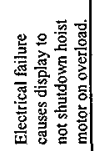 \\
\hline 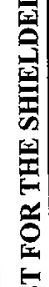 & 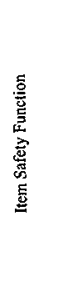 & 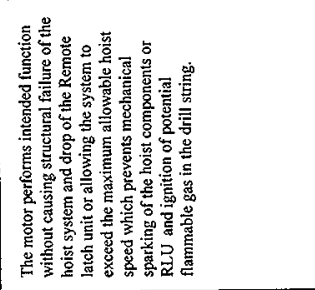 & 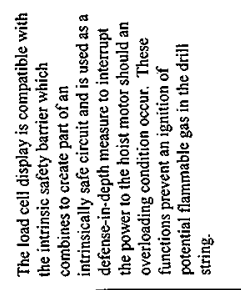 & & \\
\hline 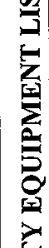 & 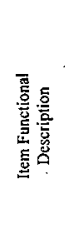 & 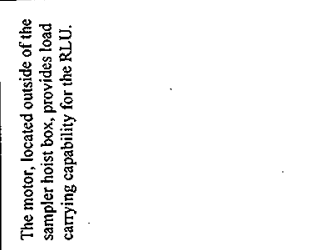 & 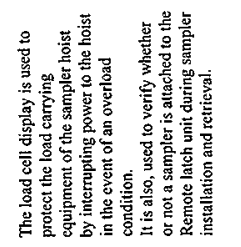 & & \\
\hline 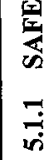 & 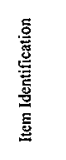 & 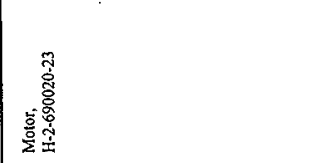 & 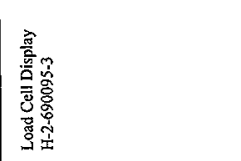 & & \\
\hline
\end{tabular}




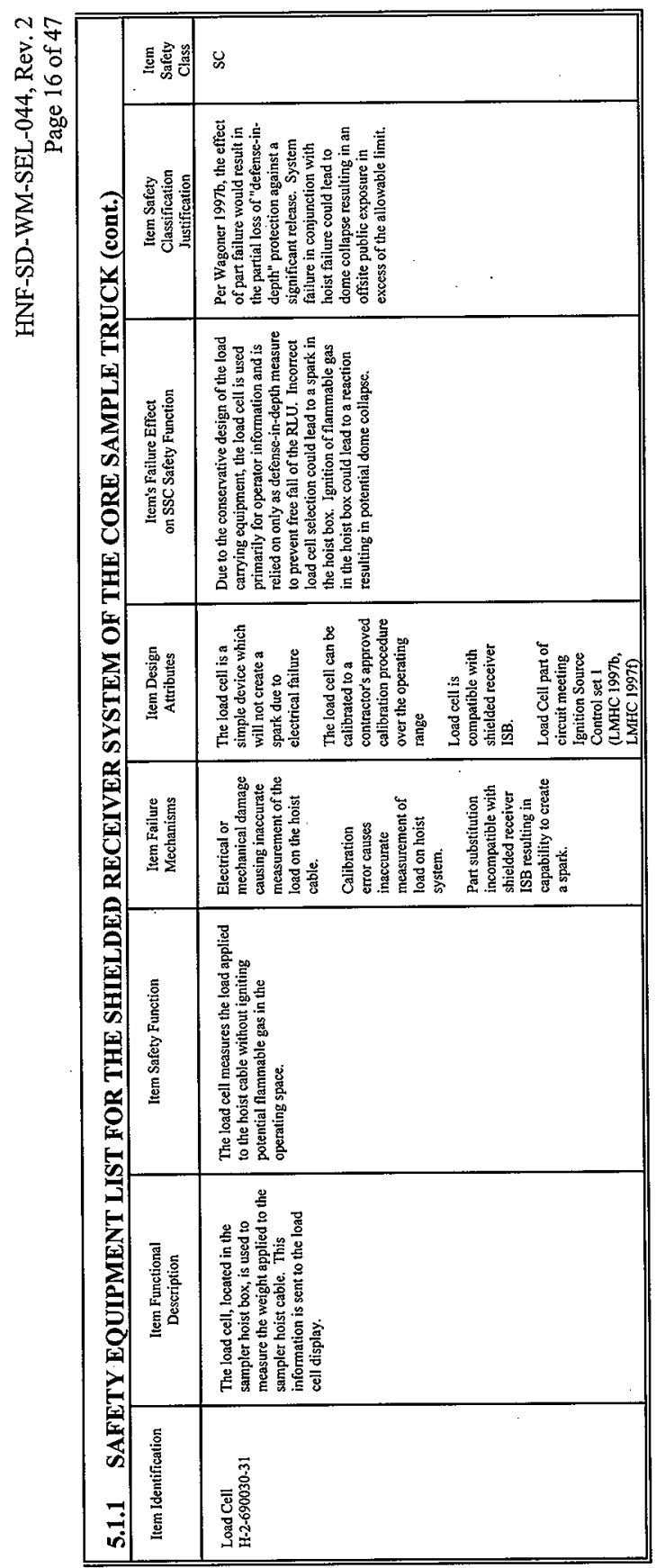




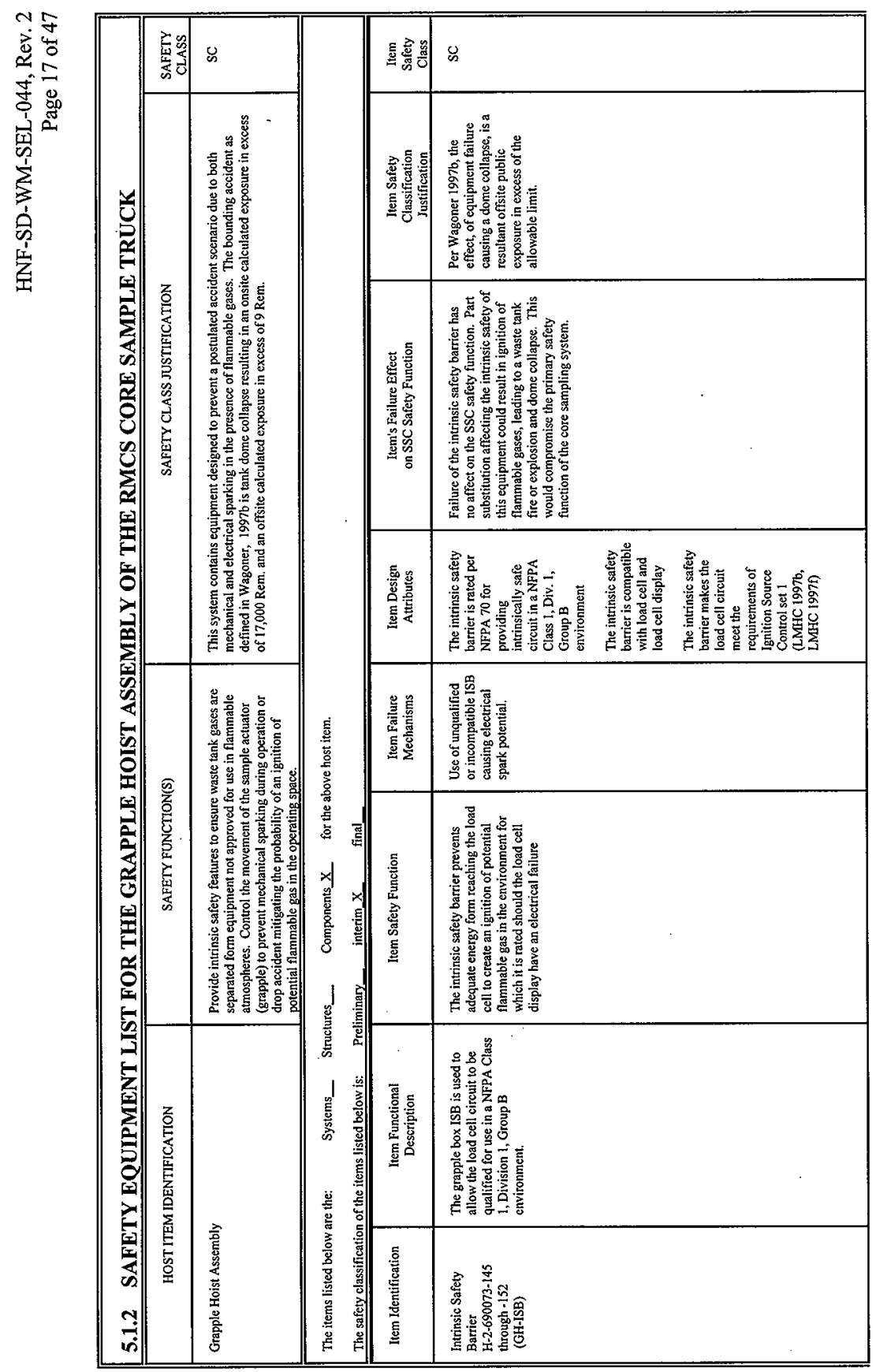




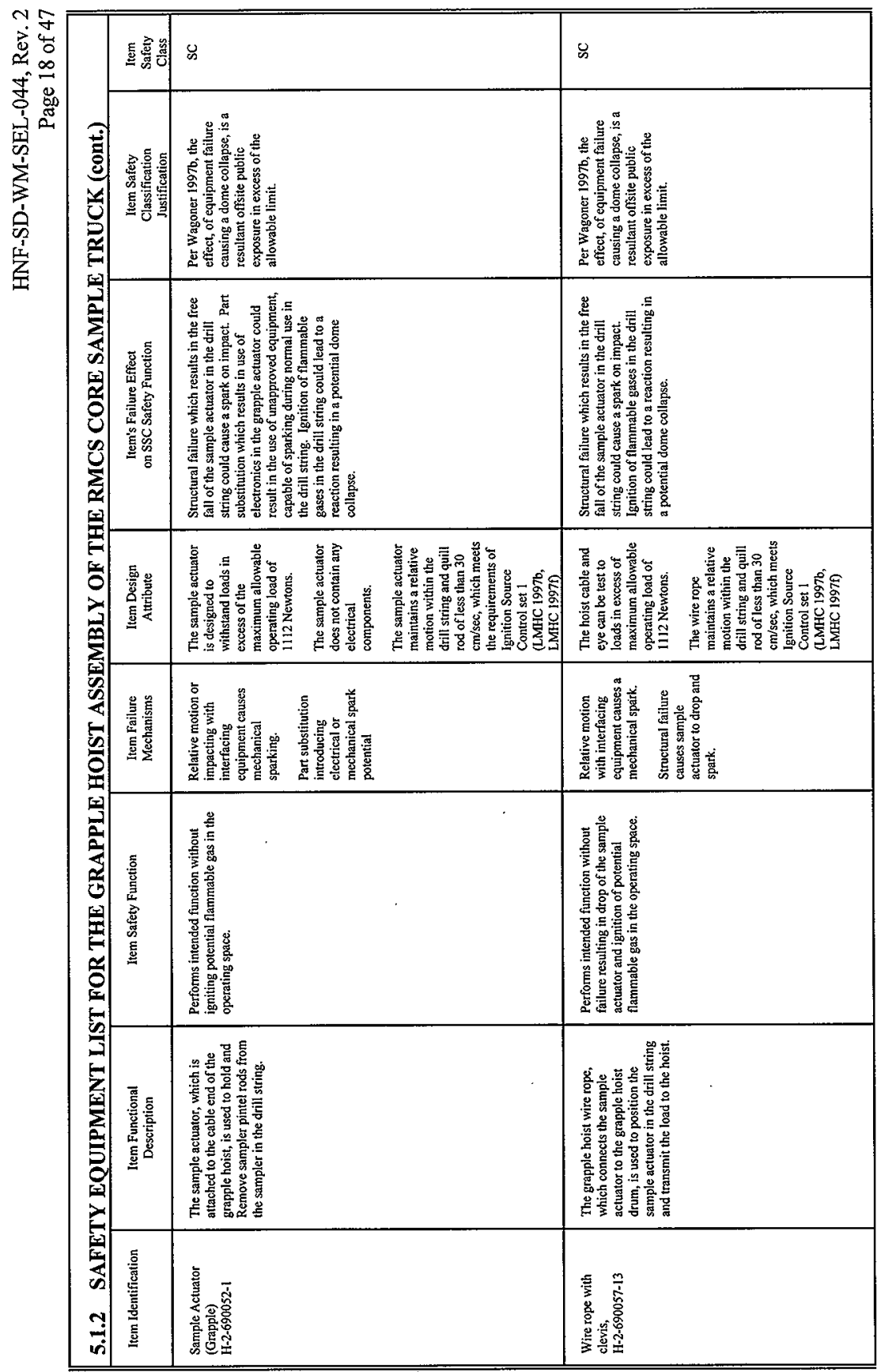




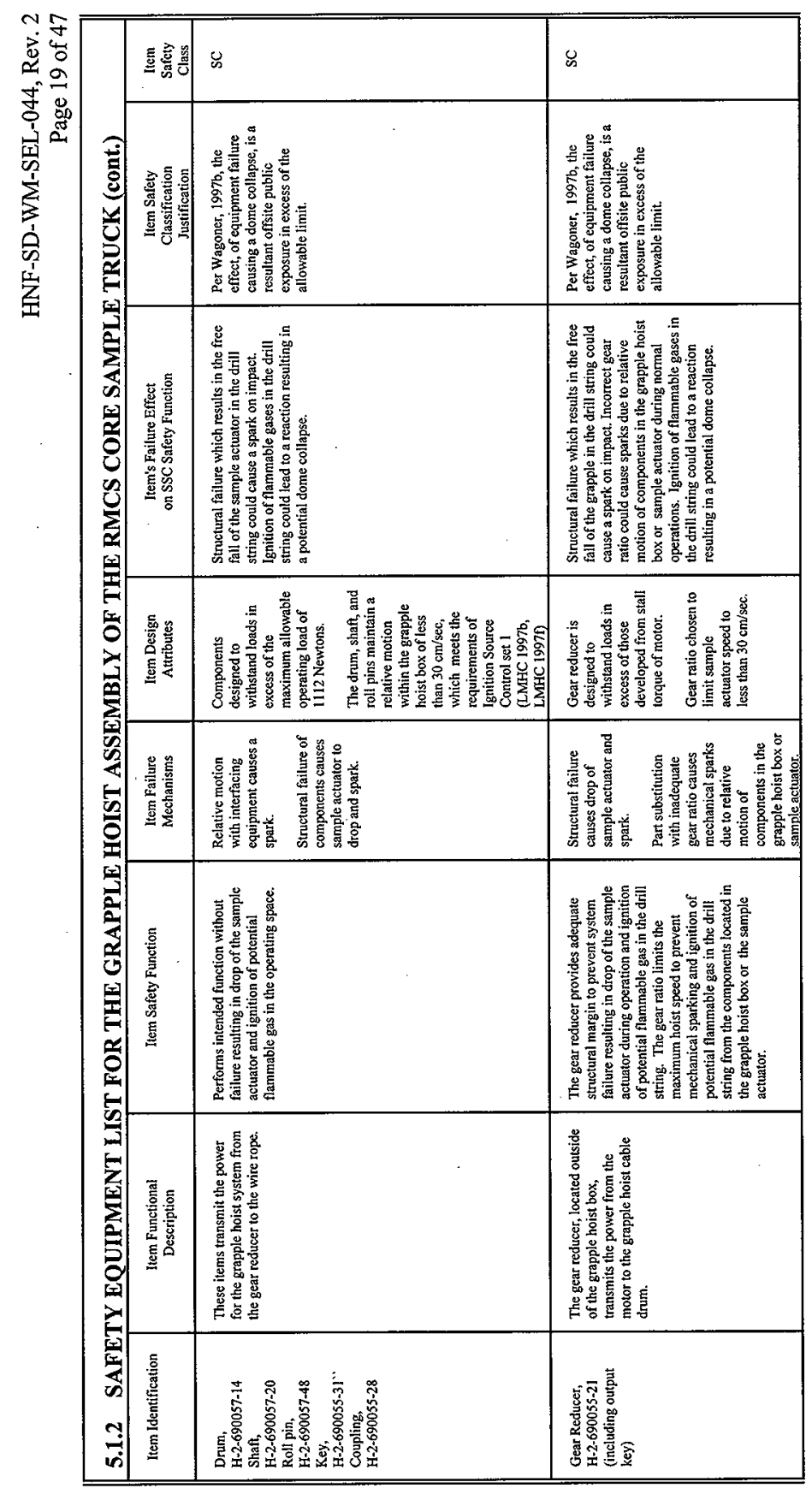




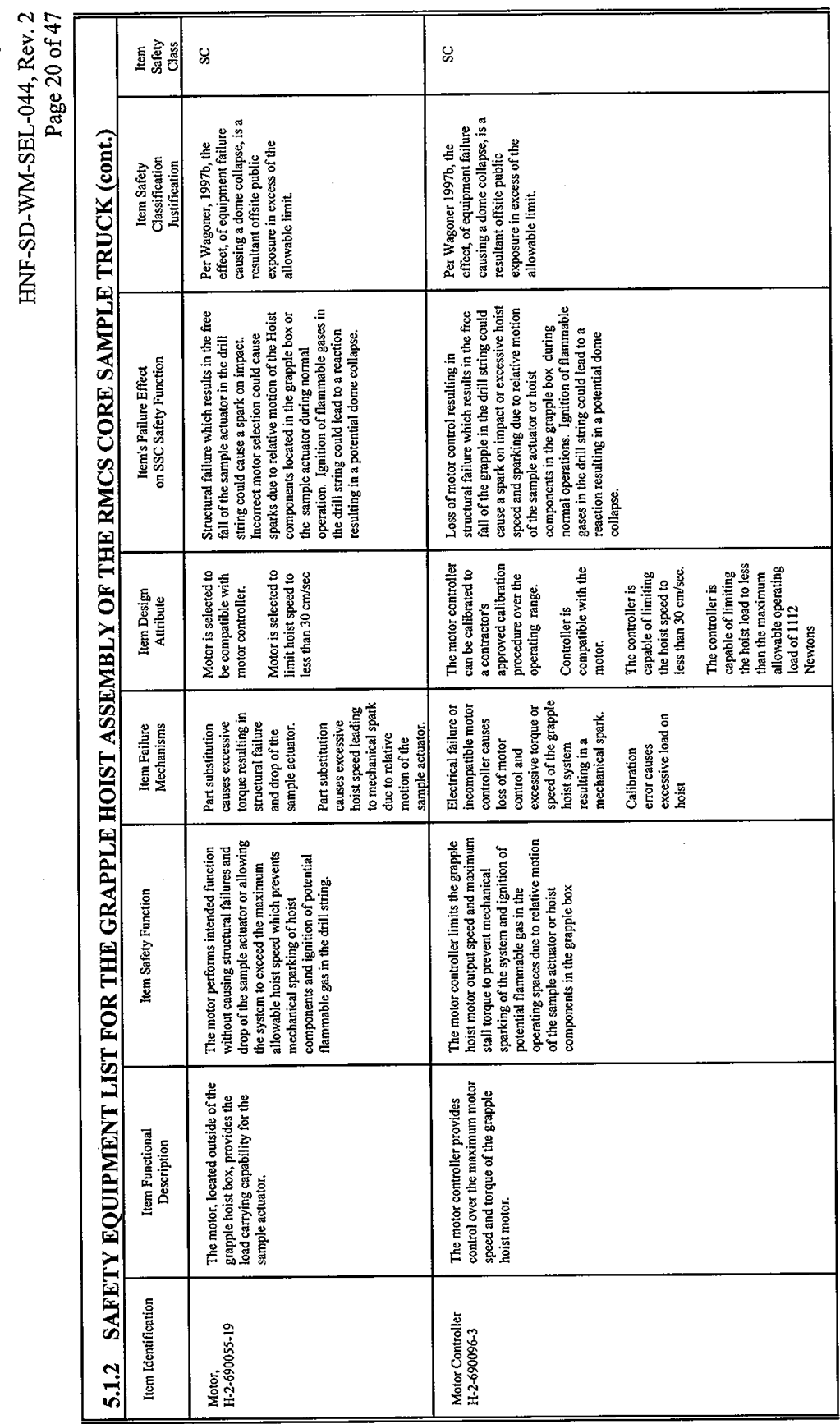




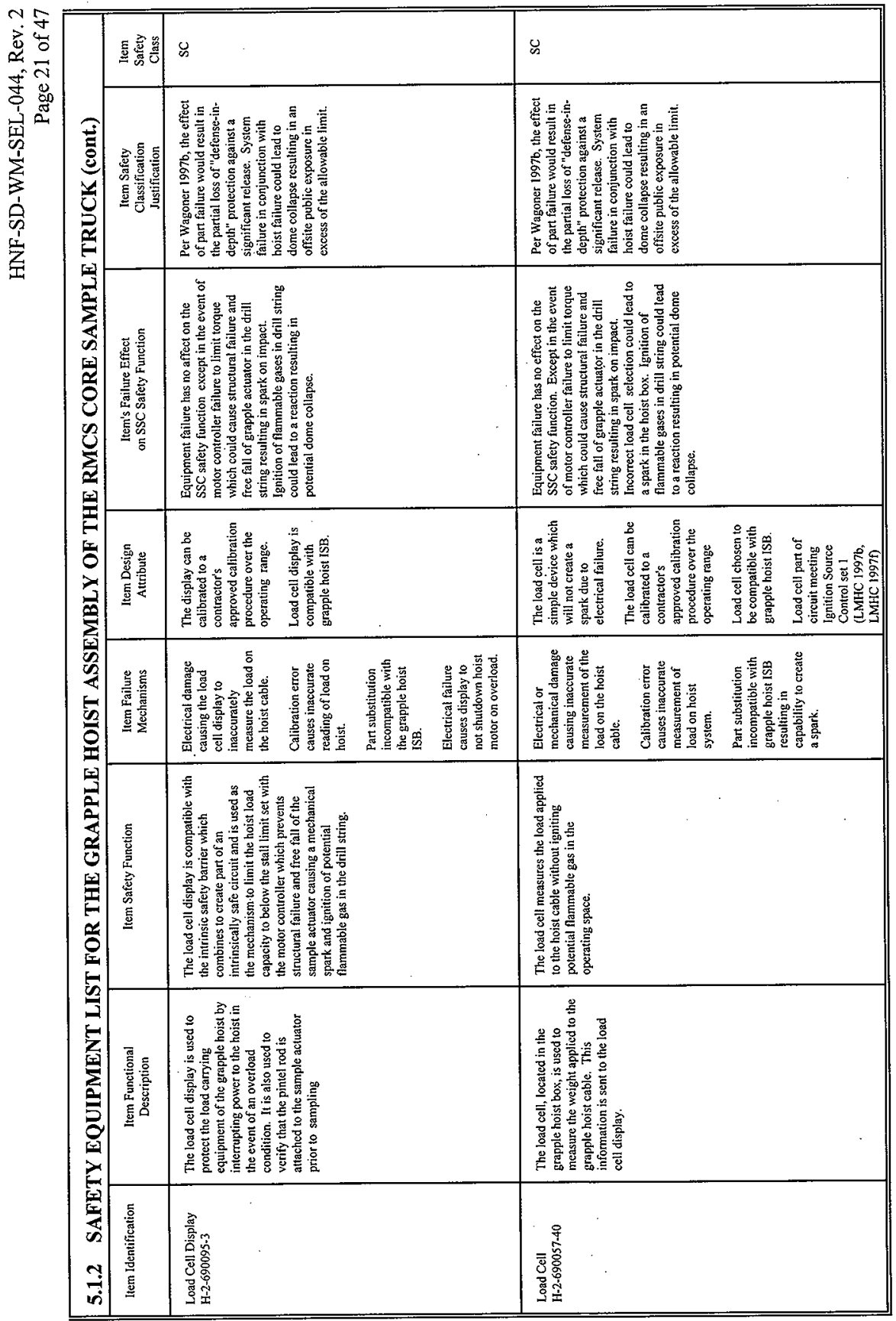




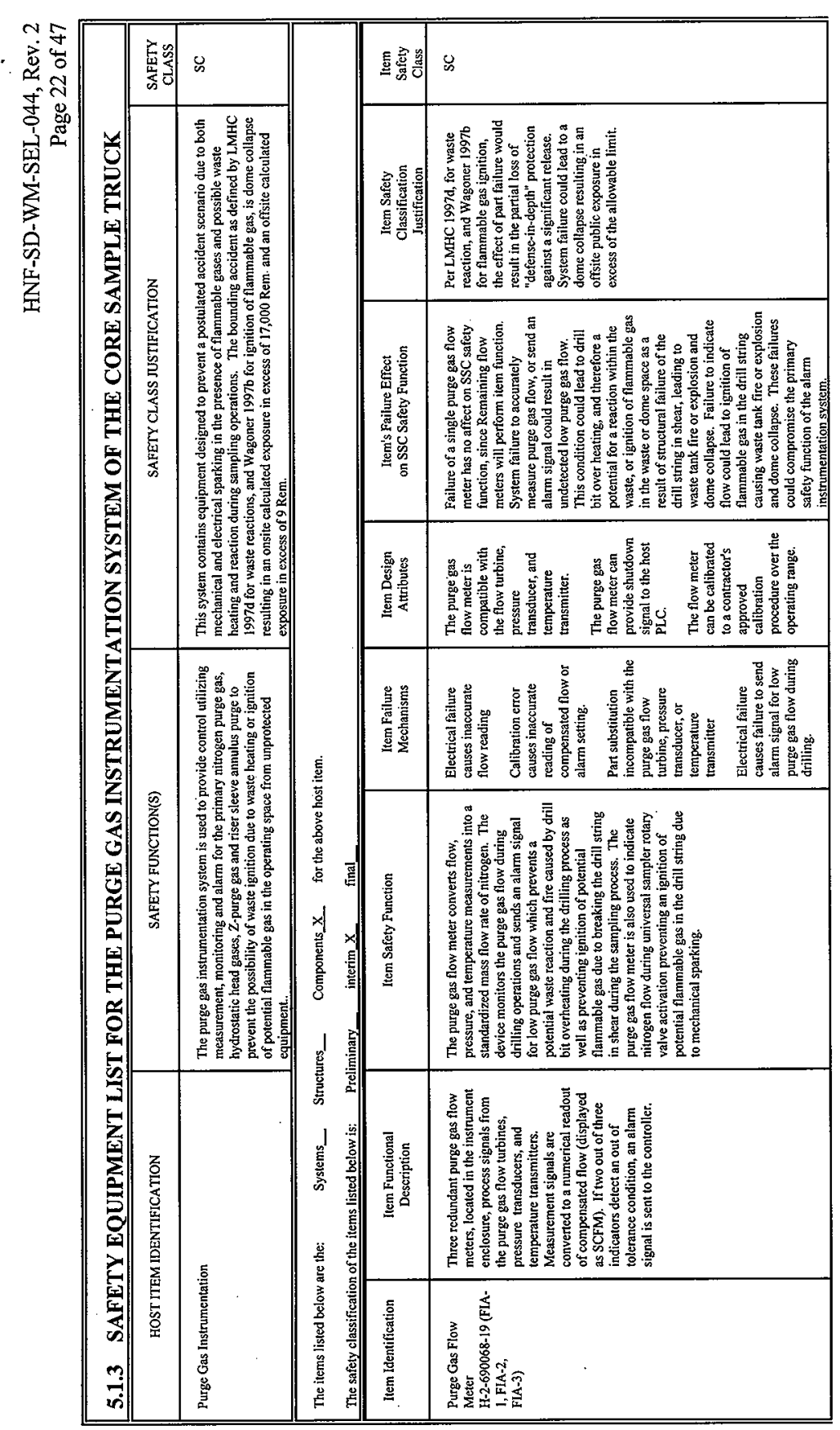




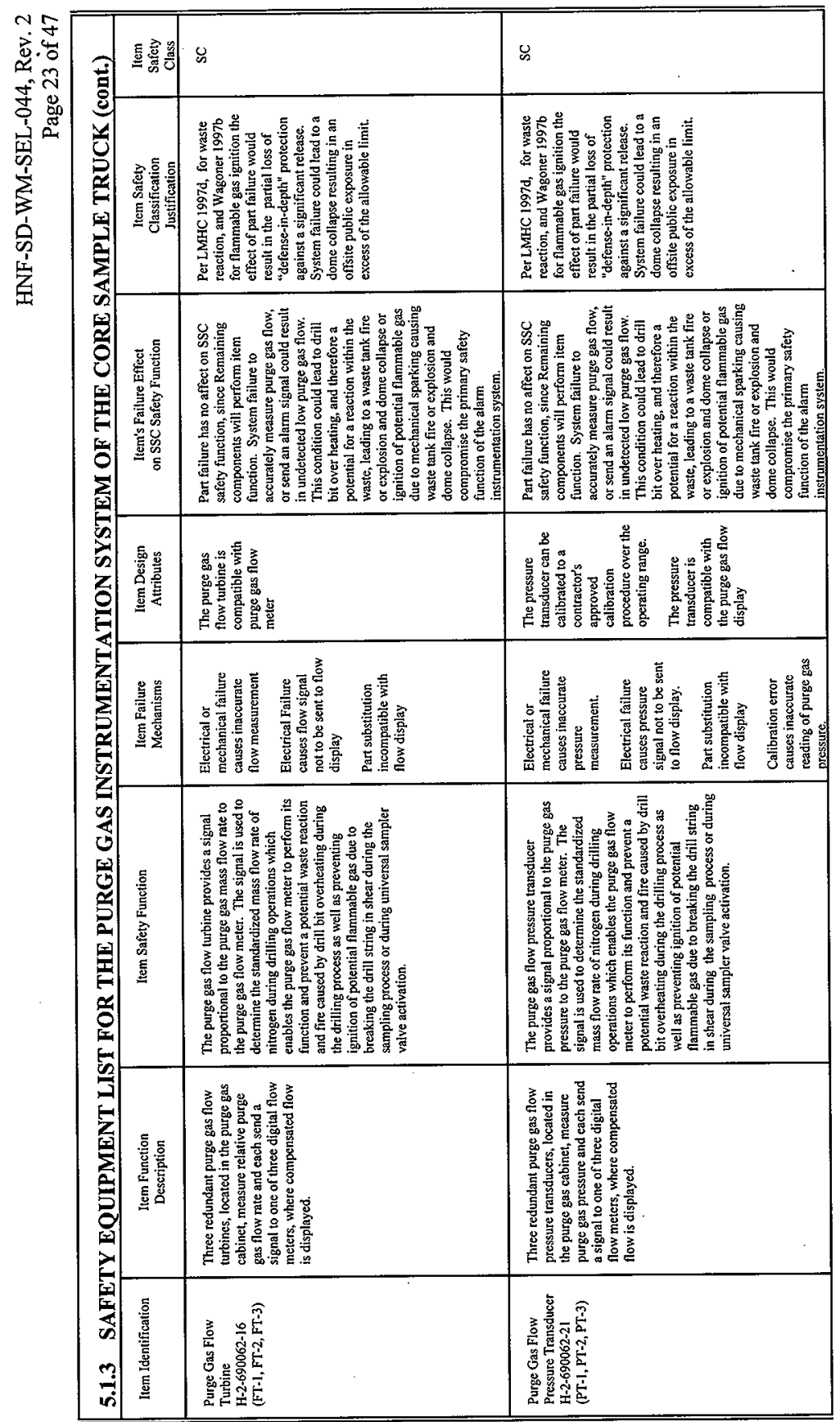




\begin{tabular}{|c|c|c|c|c|}
\hline $\mathcal{B}$ & 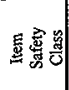 & \& & U্口 & $\mathscr{B}$ \\
\hline 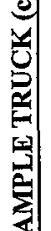 & 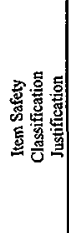 & 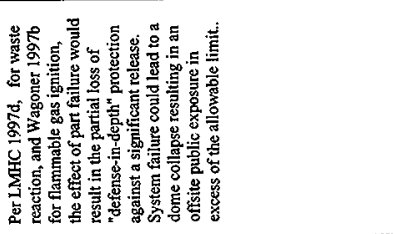 & 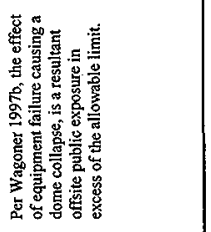 & 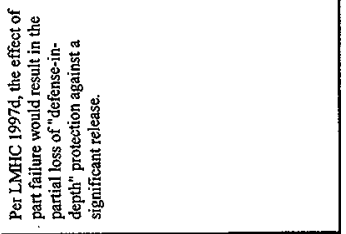 \\
\hline | & 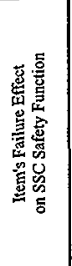 & 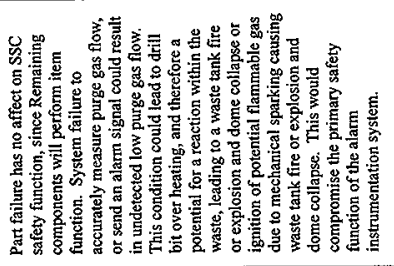 & 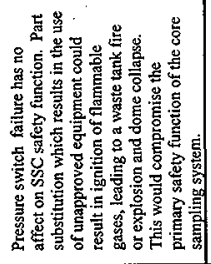 & 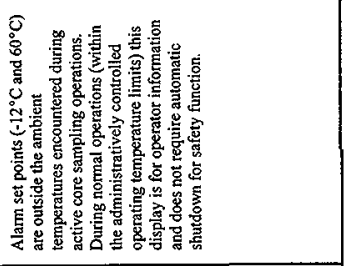 \\
\hline 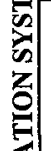 & 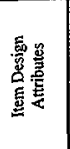 & 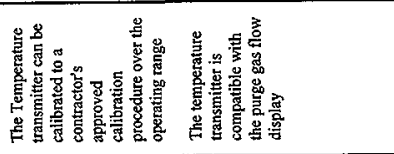 & 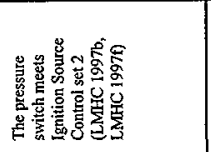 & 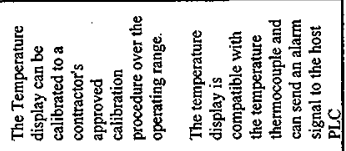 \\
\hline 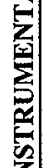 & 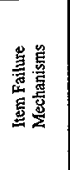 & 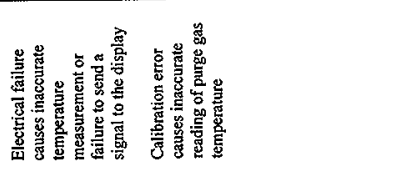 & 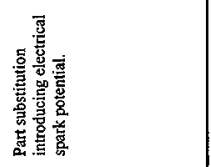 & 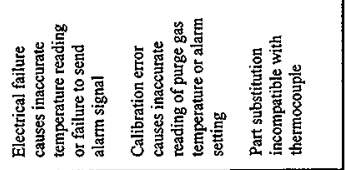 \\
\hline 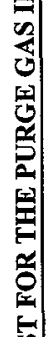 & 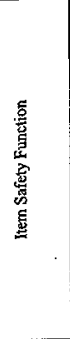 & 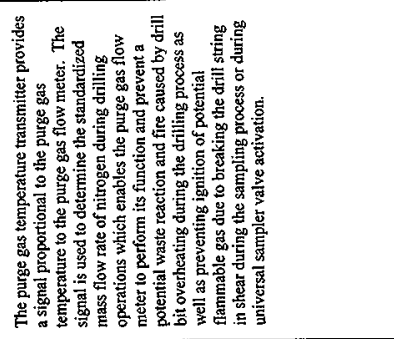 & 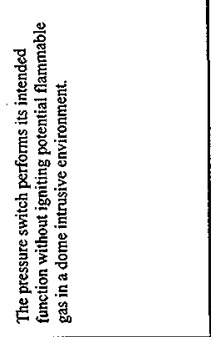 & 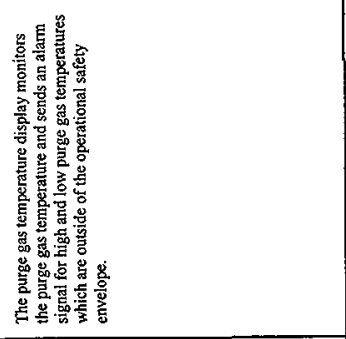 \\
\hline 国 & 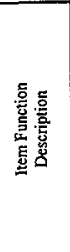 & 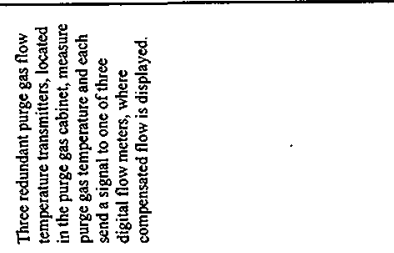 & 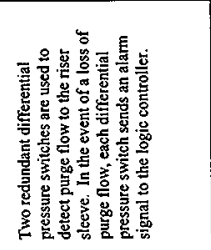 & 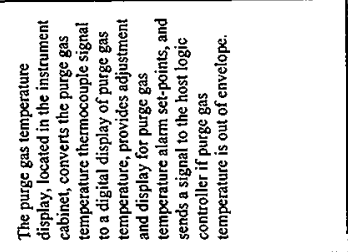 \\
\hline 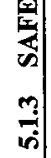 & 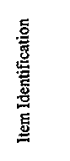 & 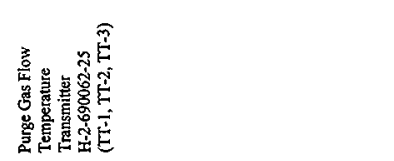 & 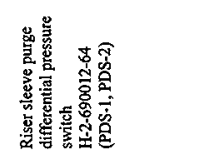 & 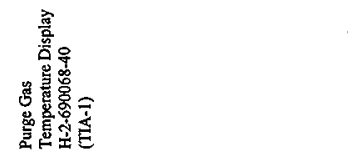 \\
\hline
\end{tabular}




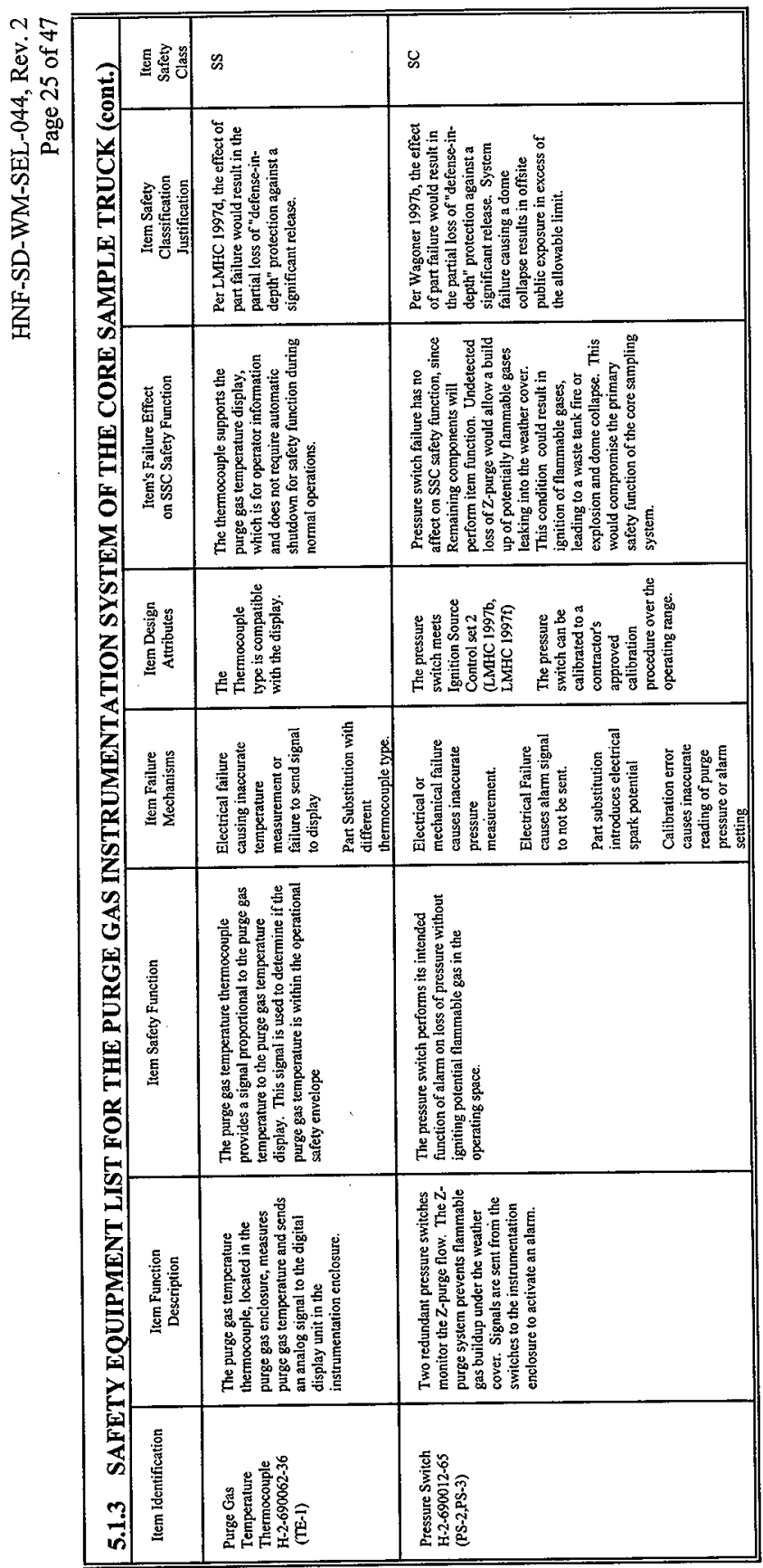




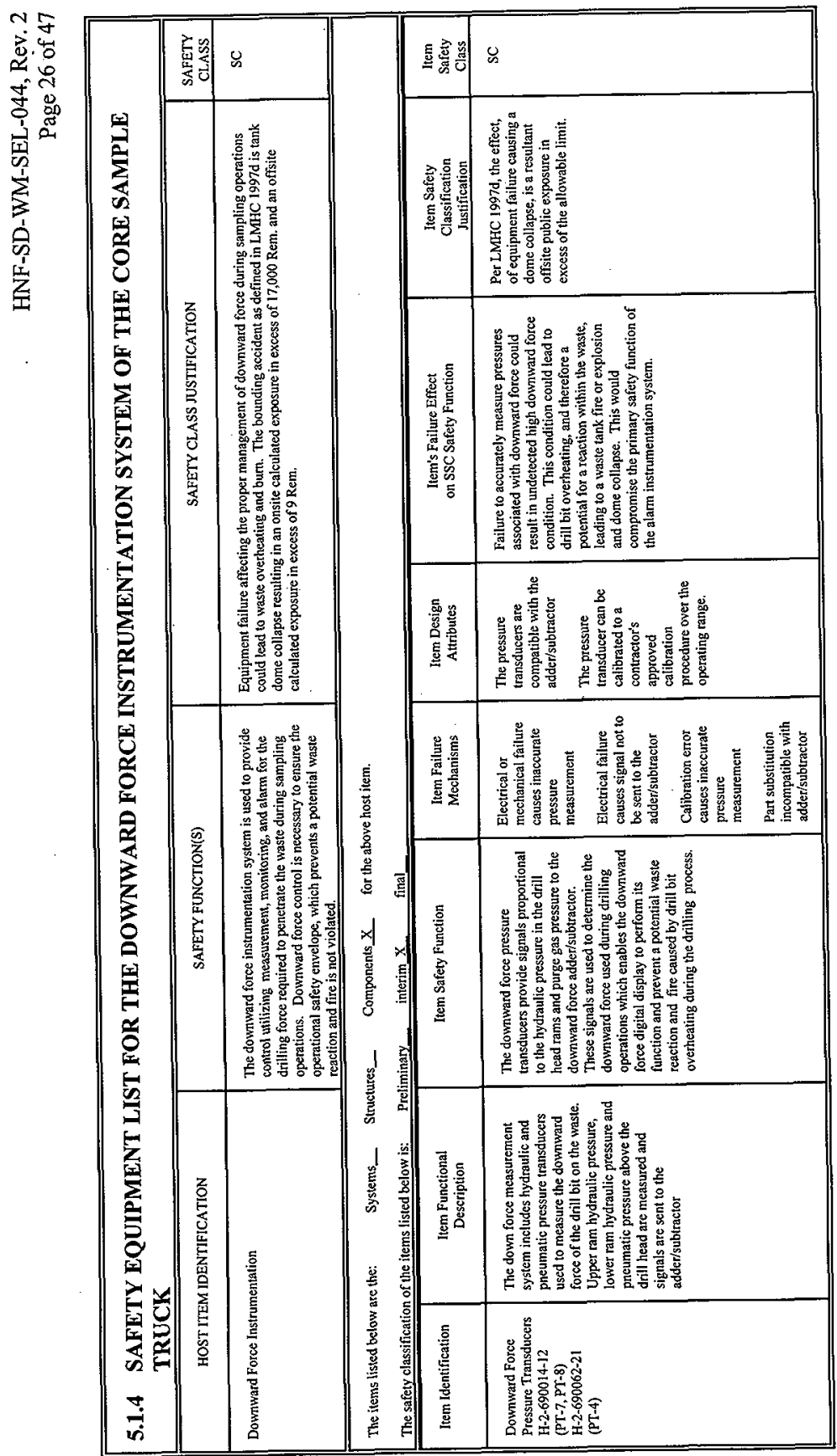




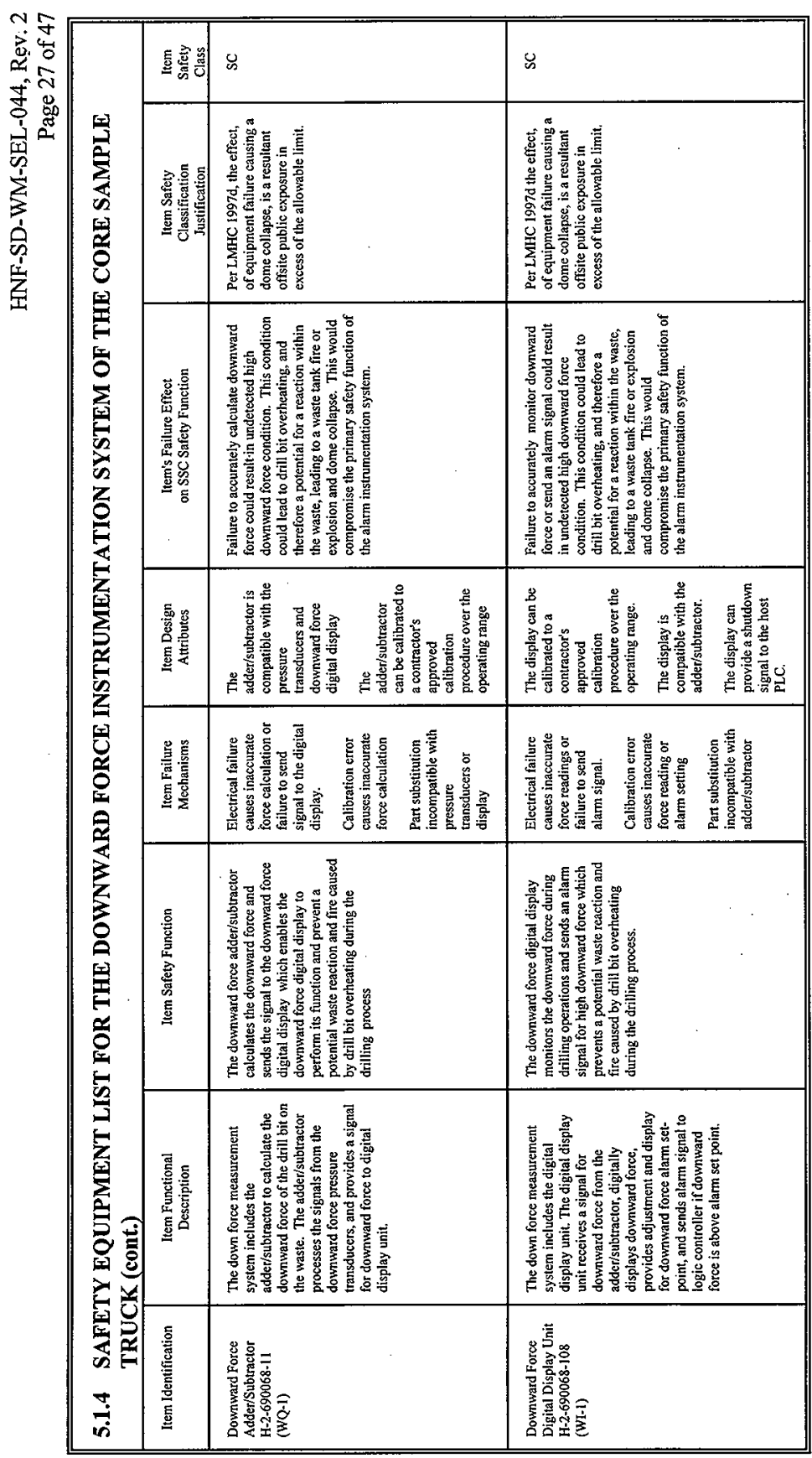




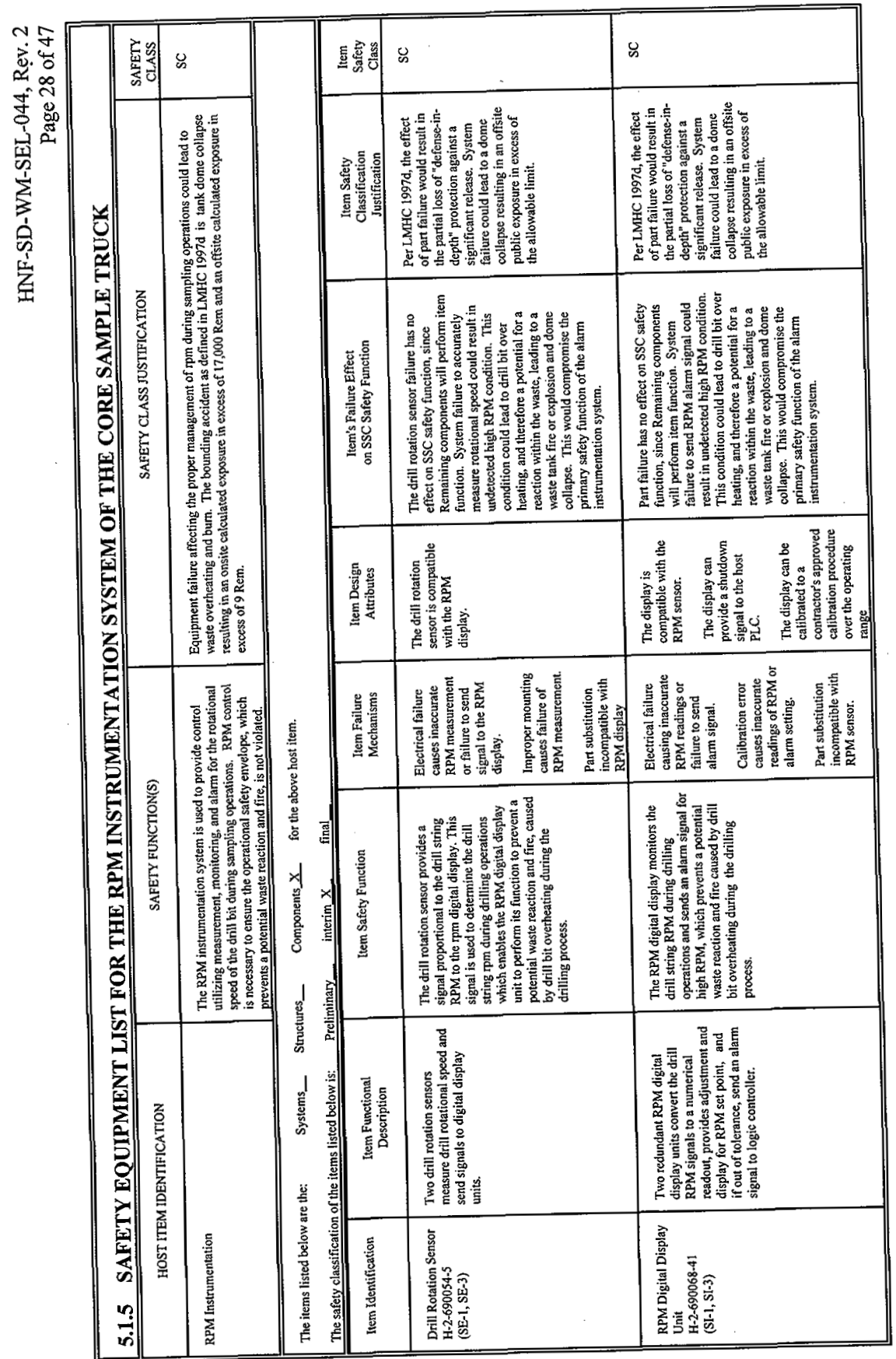




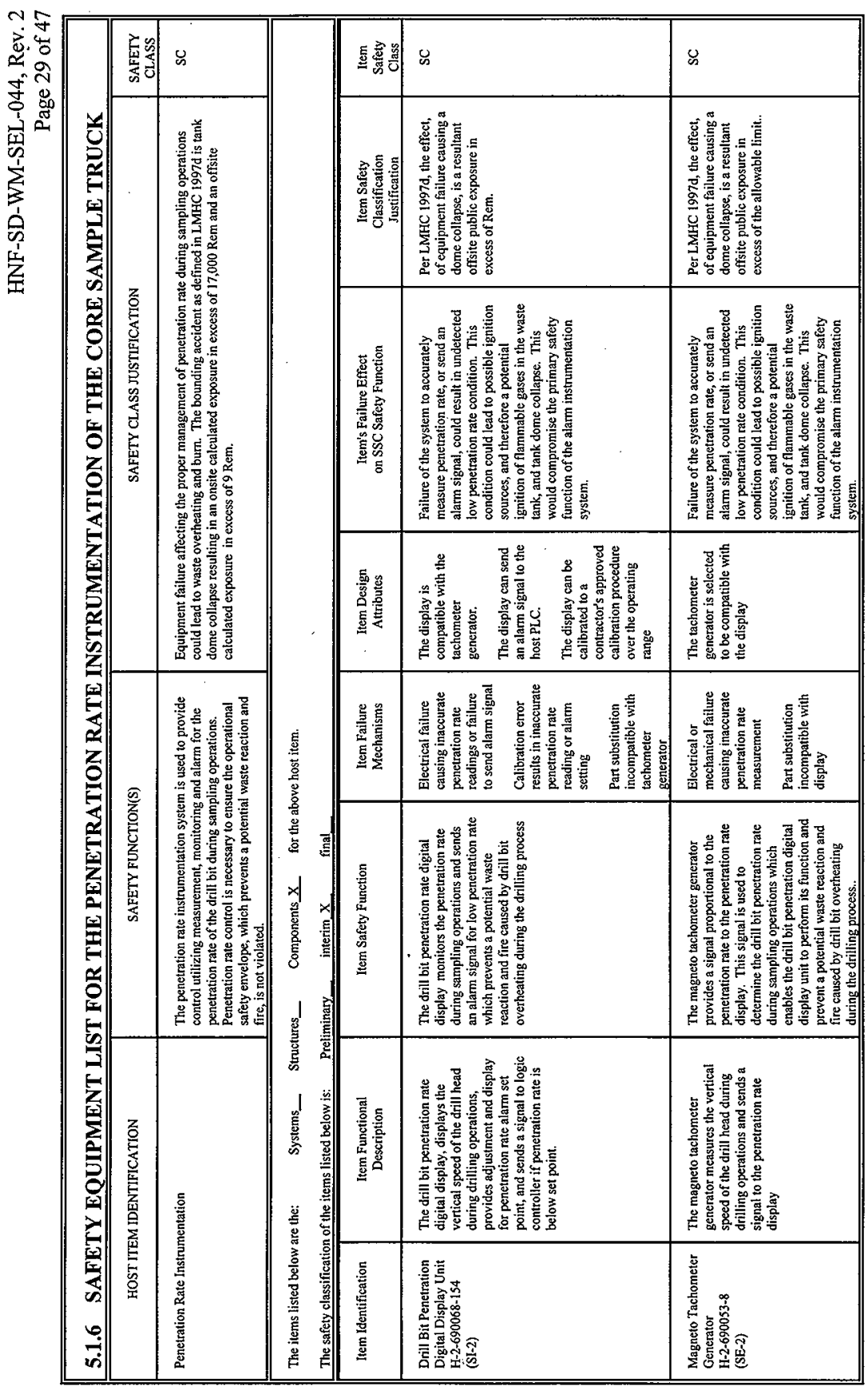




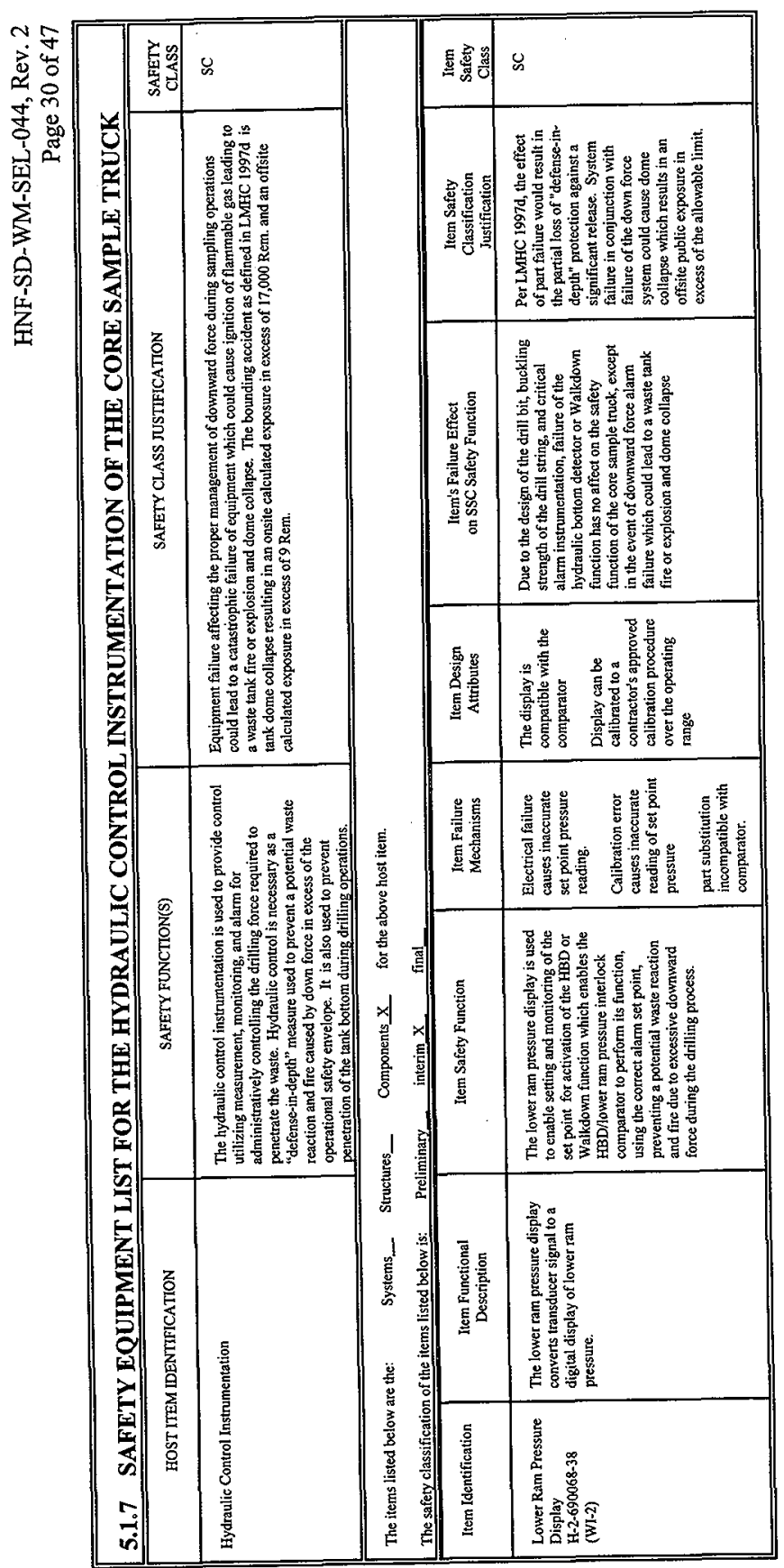




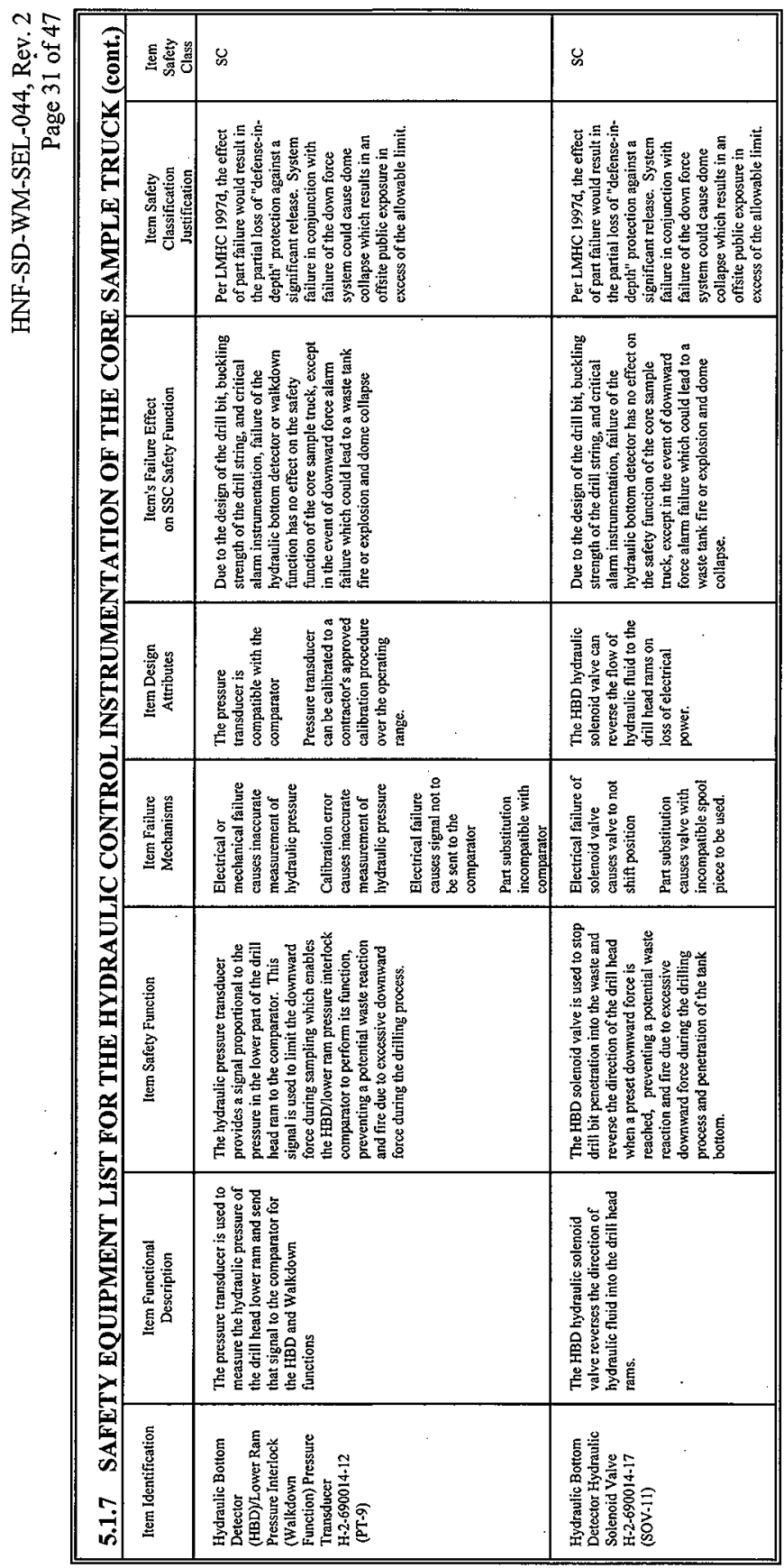




\begin{tabular}{|c|c|c|c|}
\hline$\underset{ت}{ت}$ & 屡离慈 & O & U \\
\hline | & 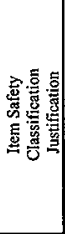 & 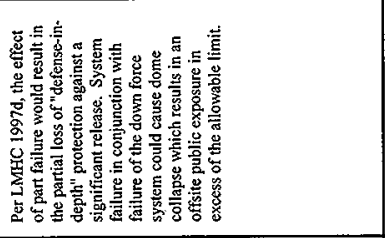 & 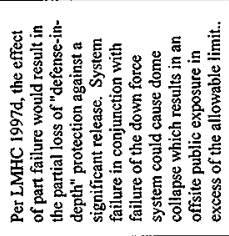 \\
\hline 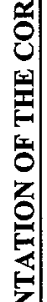 & 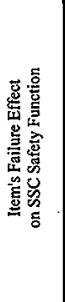 & 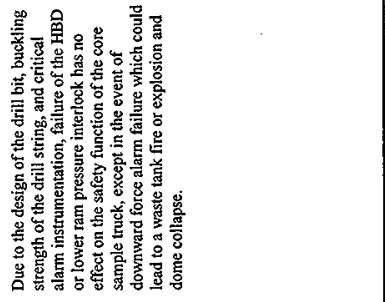 & 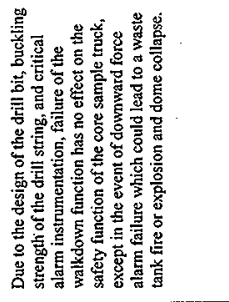 \\
\hline ב্: & 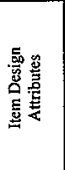 & 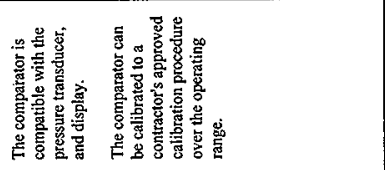 & 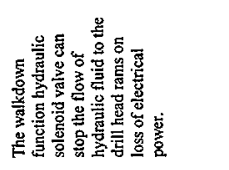 \\
\hline 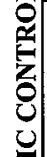 & 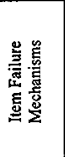 & 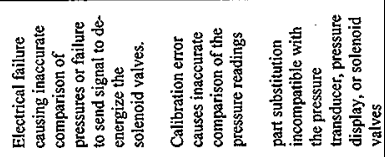 & 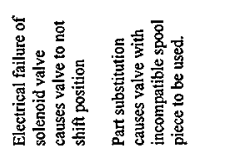 \\
\hline 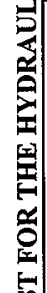 & 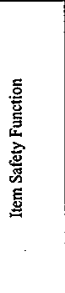 & 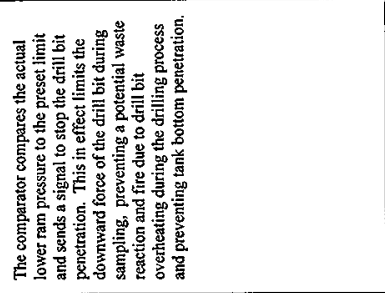 & 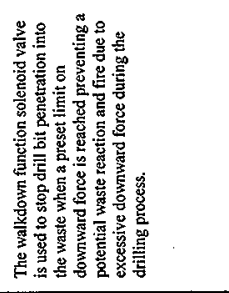 \\
\hline | & 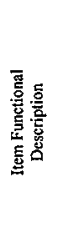 & 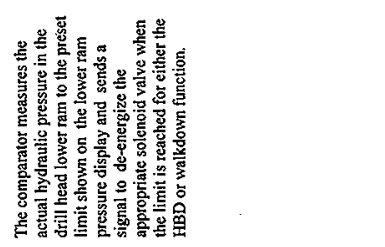 & 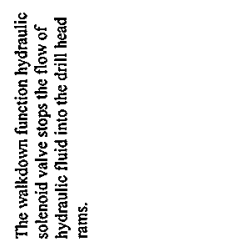 \\
\hline 乐 & 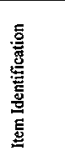 & 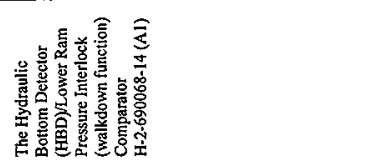 & 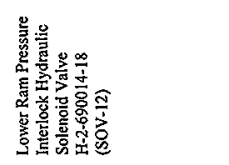 \\
\hline
\end{tabular}




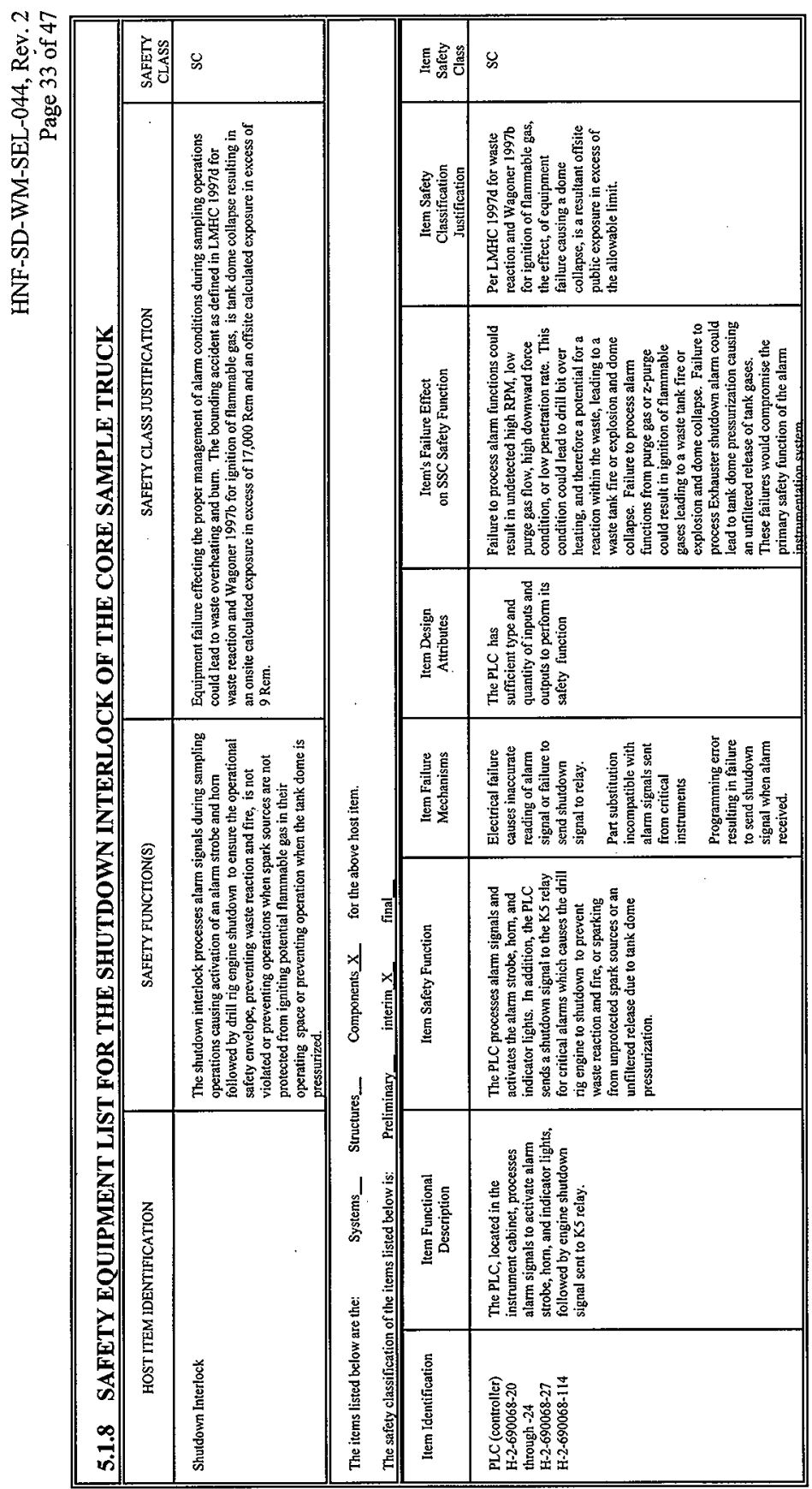




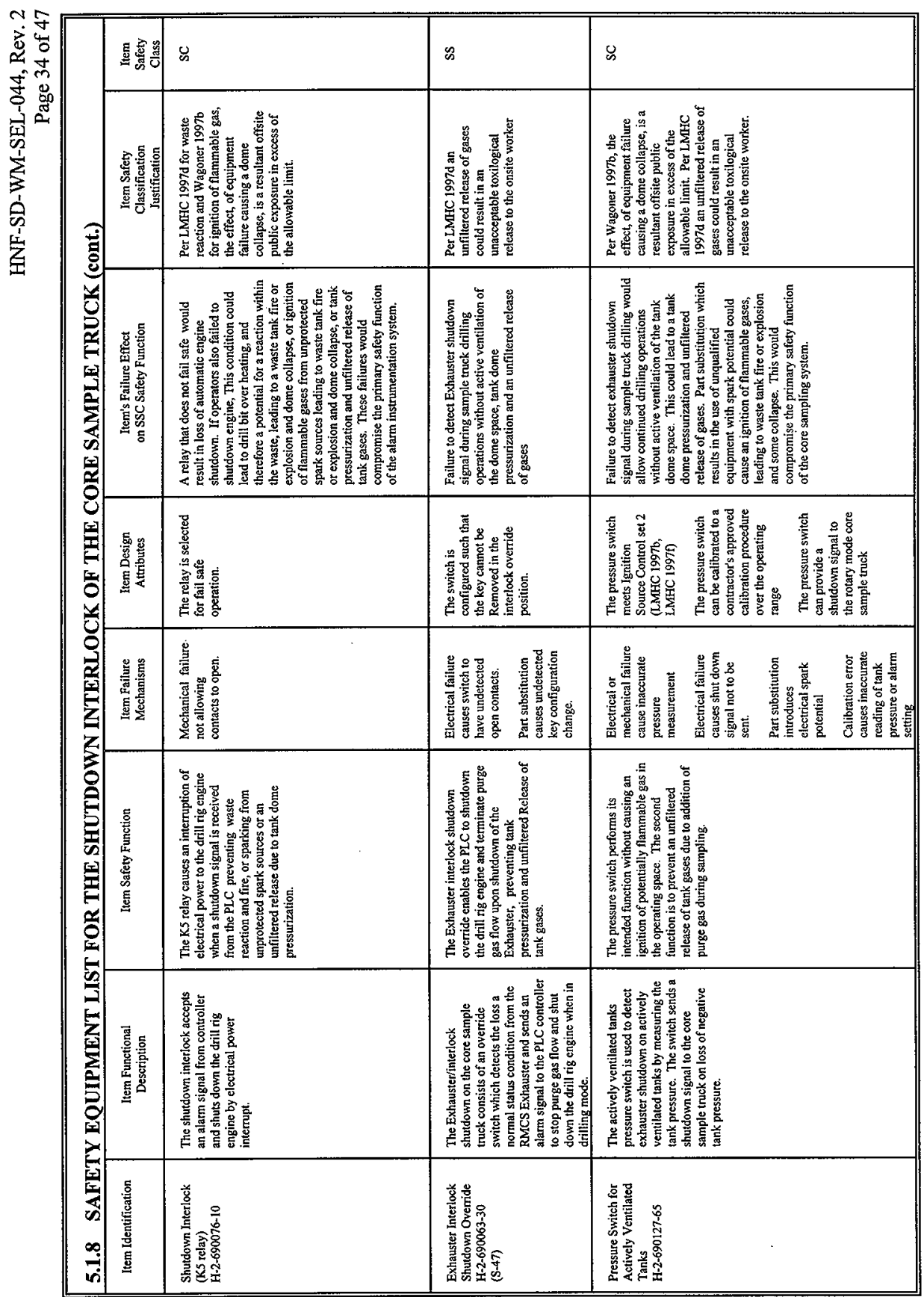




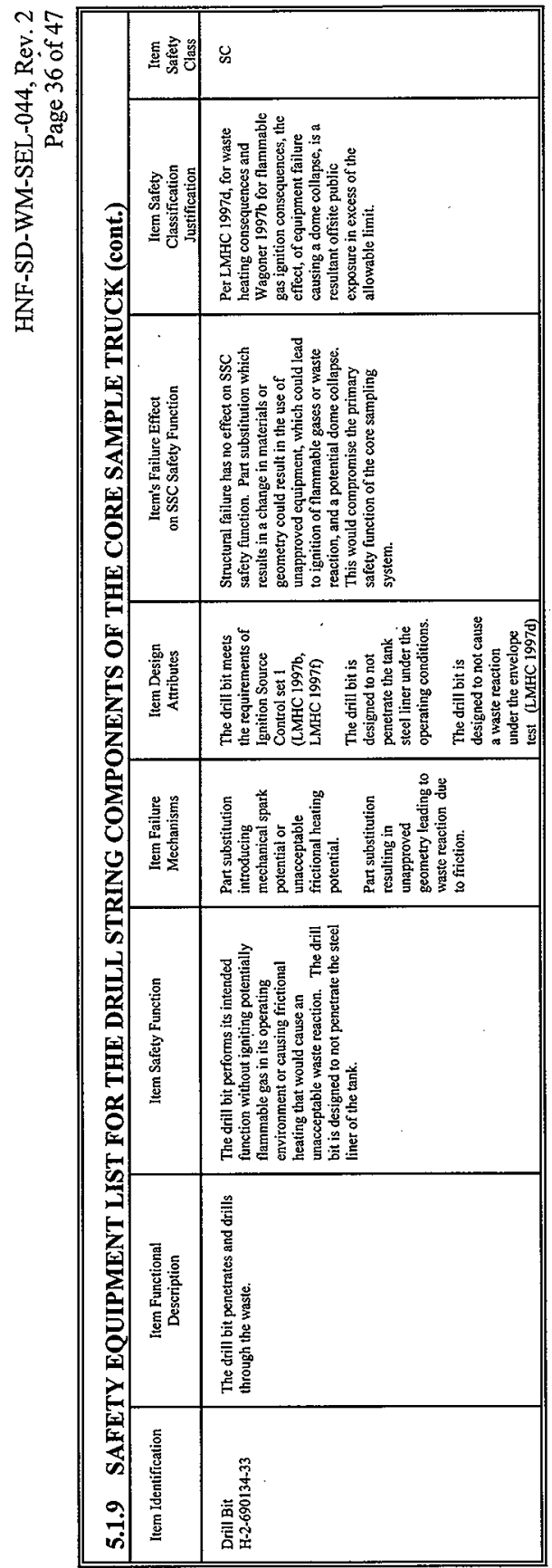




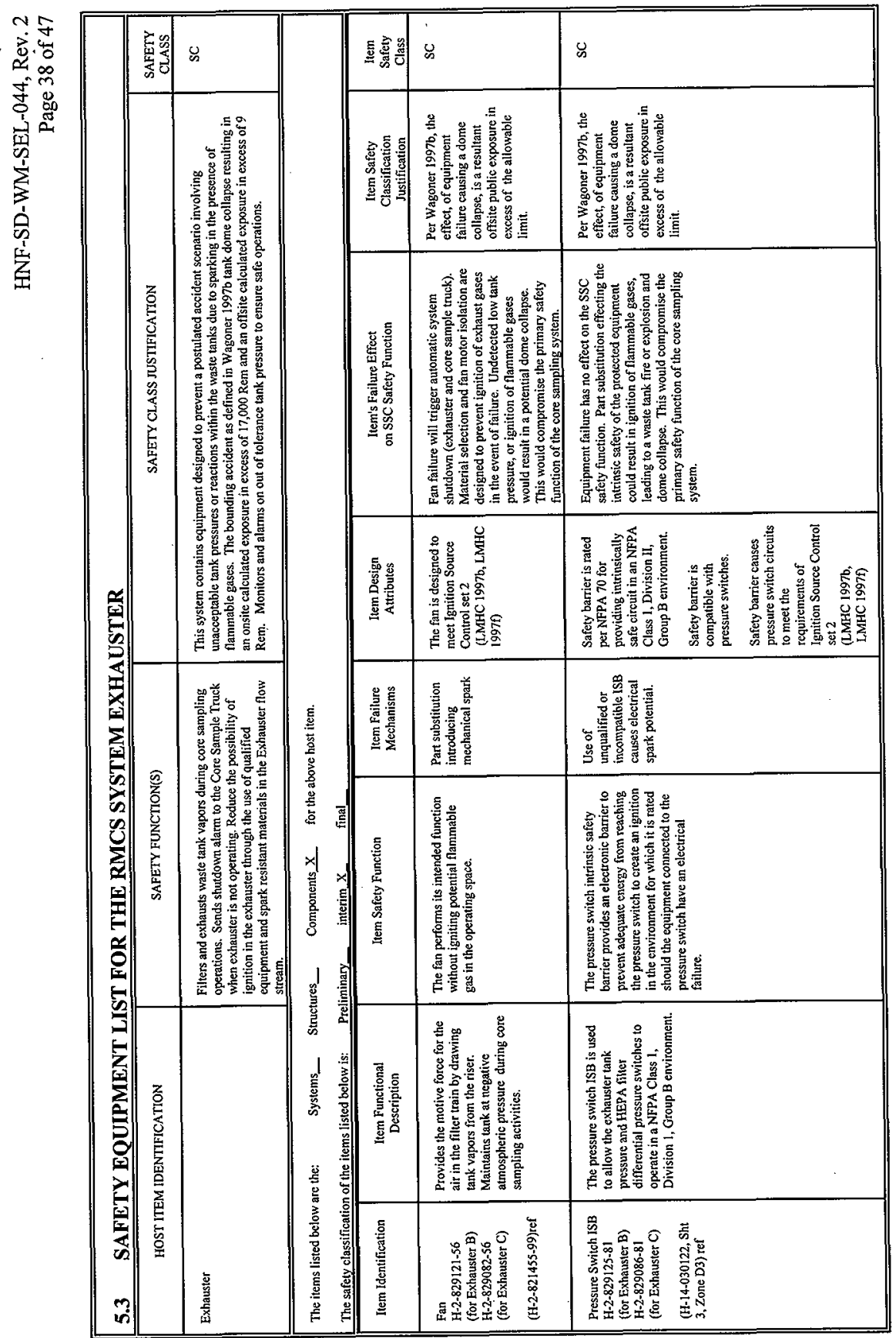




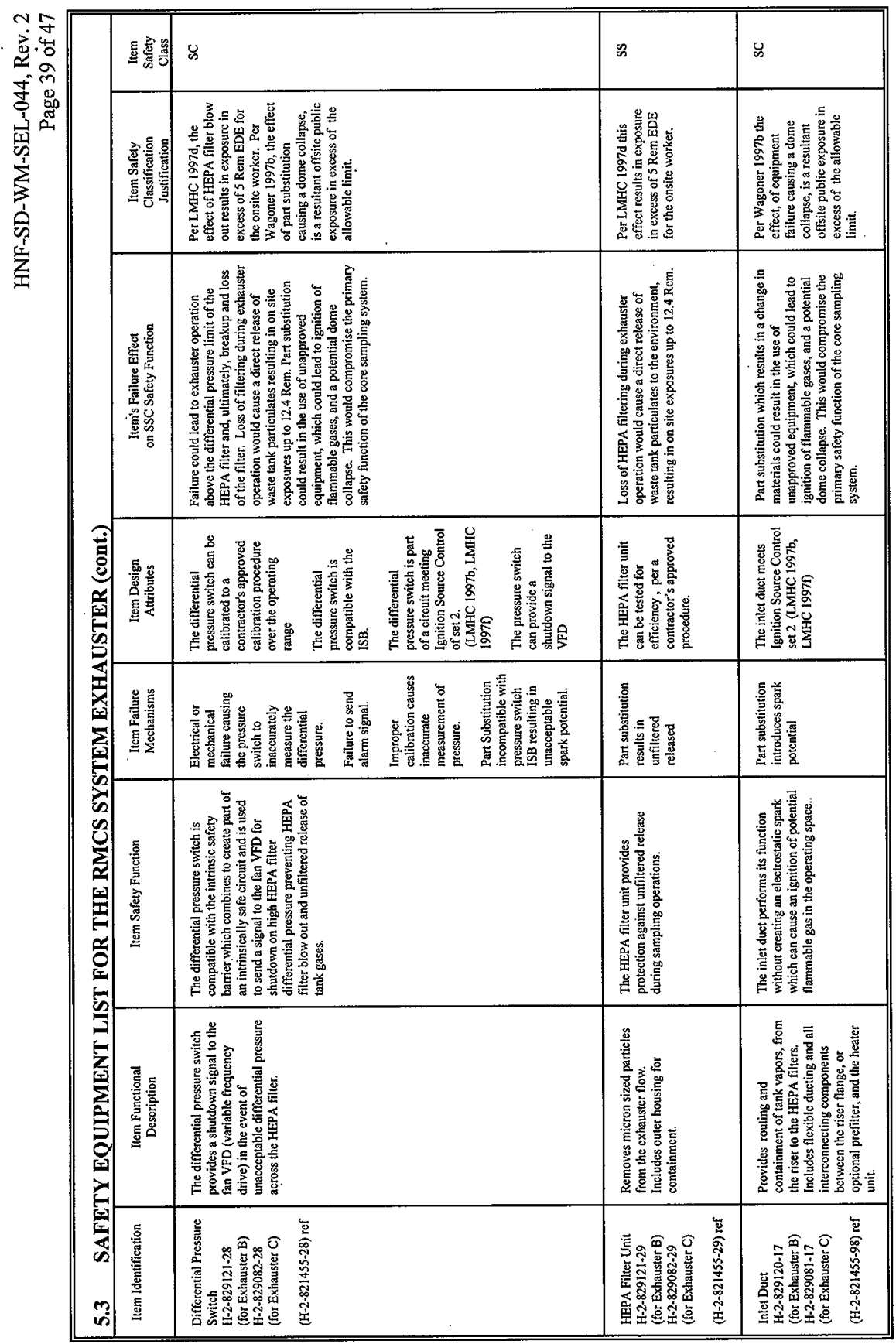




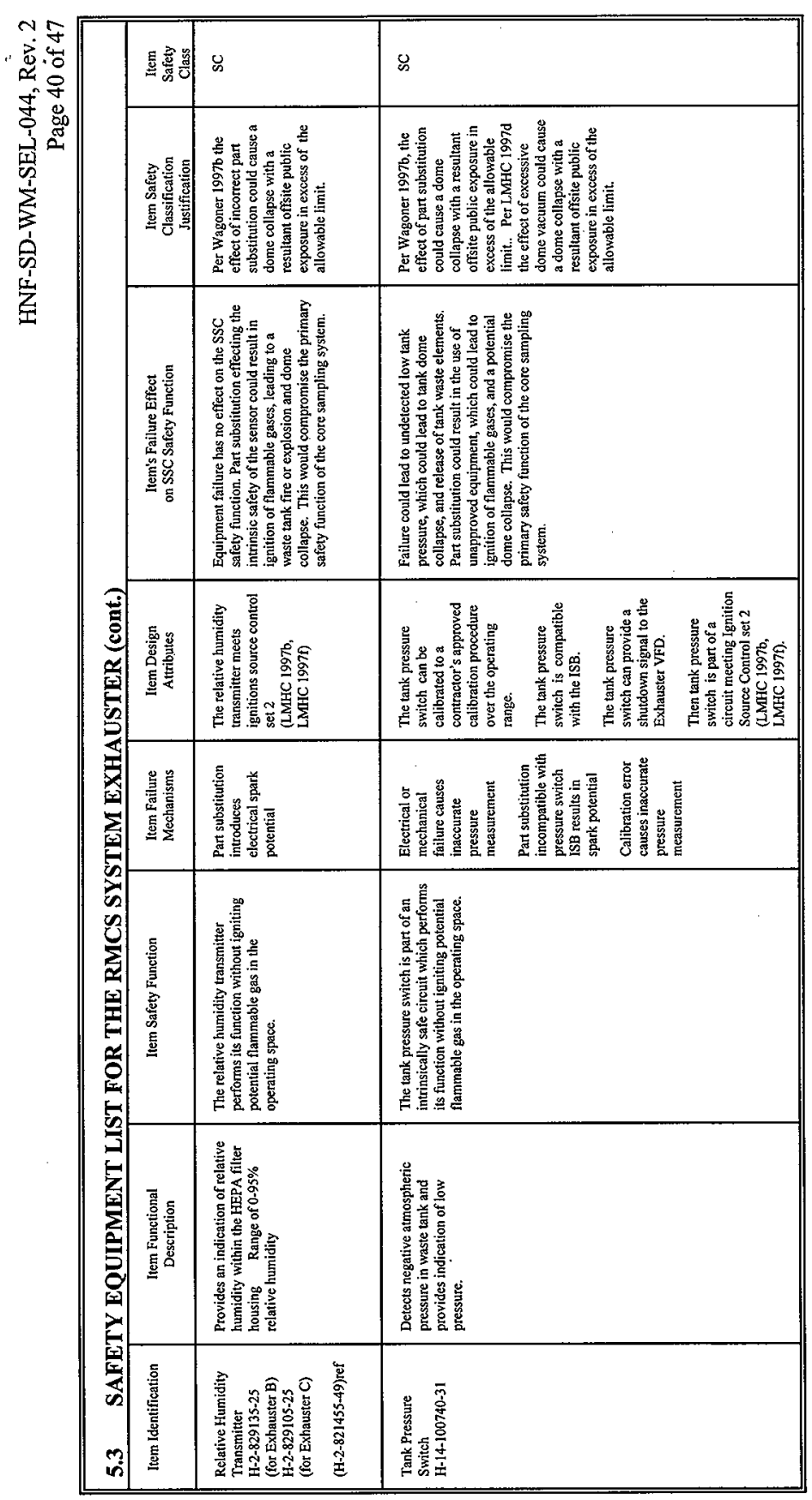




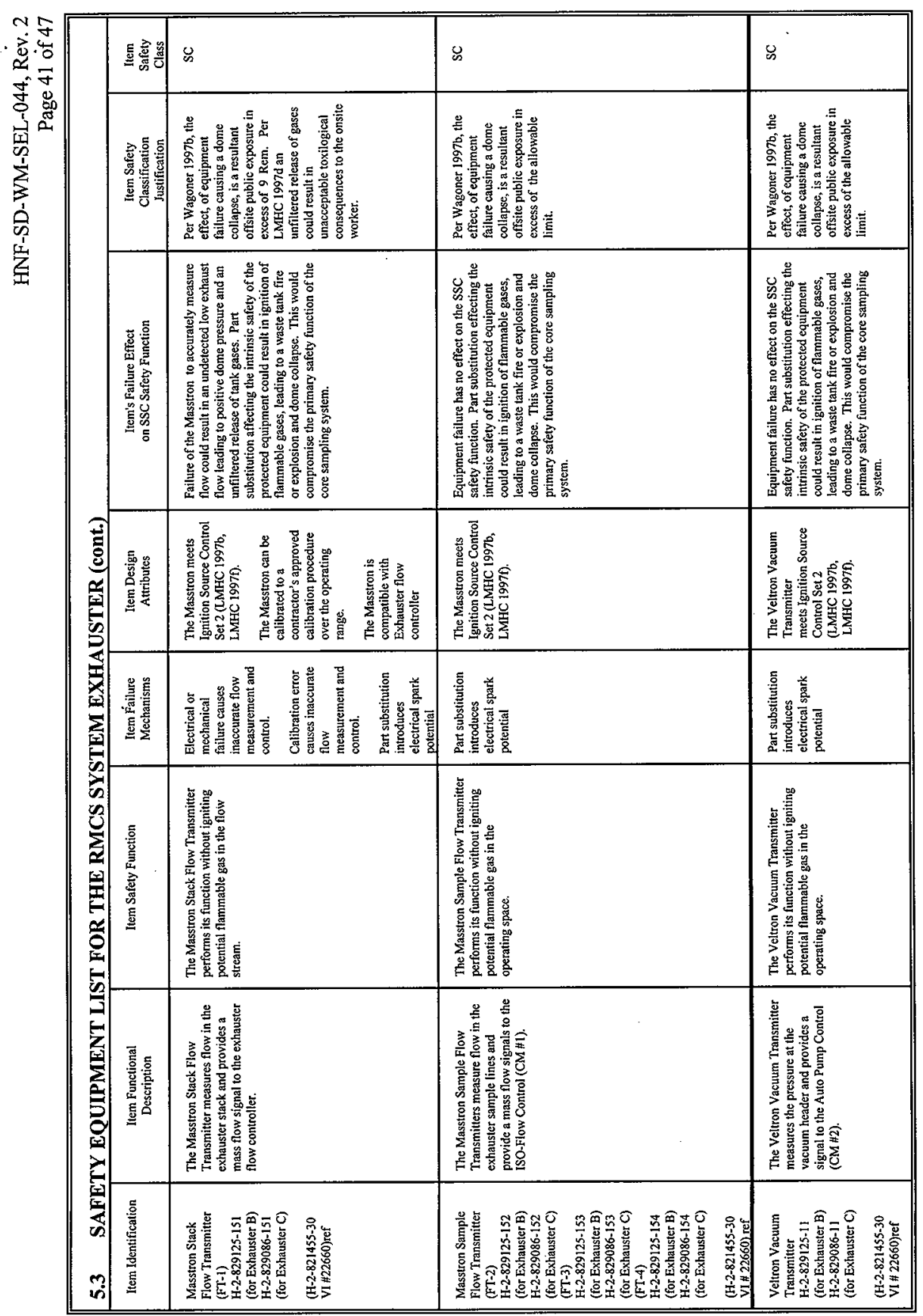




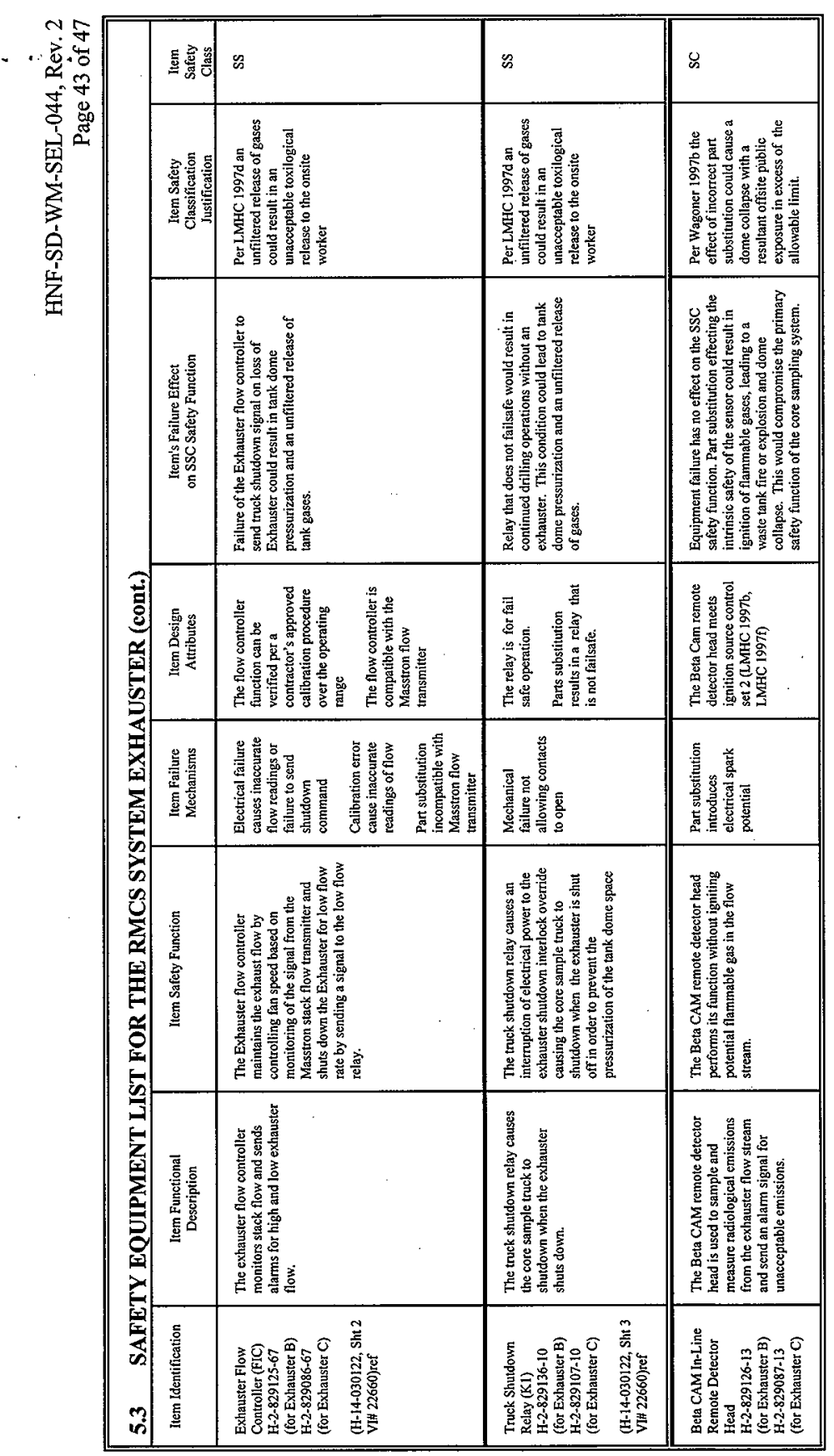




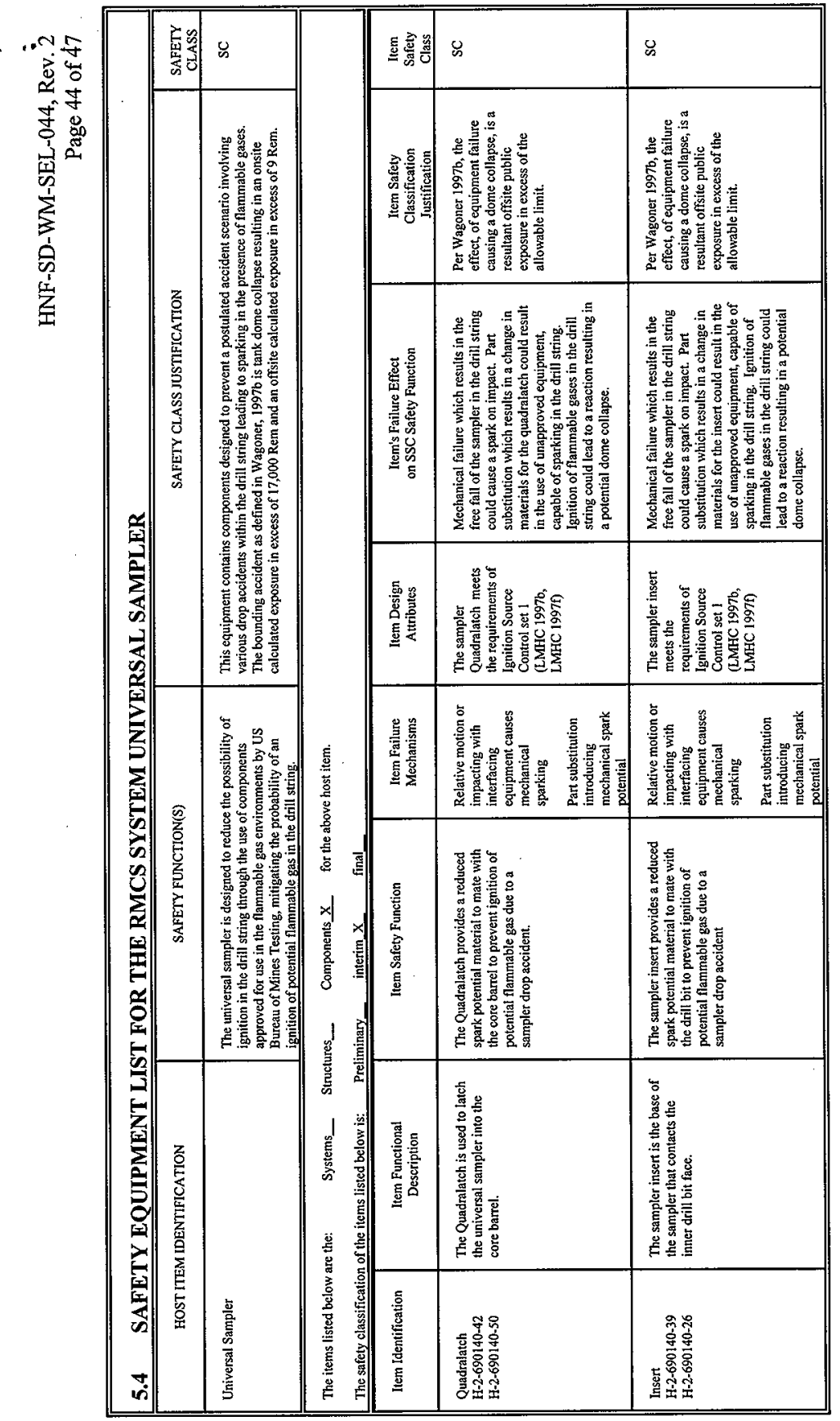




\subsection{REFERENCES}

Corbett, 1994, Rotary Mode Core Sampling Safety Equipment List, WHC-SD-WM-SEL-032, Latest Revision, Westinghouse Hanford Company, Richland, Washington.

DOE-RL, 1996 RMCSS Safety Evaluation Report, Third Tier Review Prepared for the US Department of Energy, Richland Operations, Lockheed Martin Idaho Technologies Company, July 18, 1996.

HNF 1997a, HNF-PRO-447, Revision 0, Project Hanford Policy and Procedure System, Procurement of Safety Class Items and Management of Spares, Fluor Daniel Hanford Company, Richland, Washington.

HNF 1997b, HNF-PRO-516, Revision 0, Project Hanford Policy and Procedure System, Safety Structures, Systems, and Components, Fluor Daniel Hanford Company, Richland, Washington.

Kelly, 1991, WHC-SD-WM-SEL-023, Latest Revision, Safety Classification of Special Equipment for Core Sampling Hydrogen Gas and FeCN Watch List Tanks, Westinghouse Hanford Company, Richland, Washington, December, 1991.

LANL, 1996, WHC-SD-WM-SAD-035 Revision 0a, A Safety Assessment of Rotary-Mode Core Sampling in Flammable-Gas Single-Shell Tanks: Hanford Site, Richland, Washington, Westinghouse Hanford Company, Richland, Washington.

LMHC, 1996, WHC-SD-WM-OSR-005 Revision O-F, Single Shell Tank Interim Operational Safety Requirements, Lockheed Martin Hanford Corporation, Richland, Washington.

LMHC, 1997a, East and West Tank Farms Standing Order 97-01, Revision 2, Compensatory Actions for Open Discovery Unreviewed Safety Questions (USQS), Lockheed Martin Hanford Corporation, Richland, Washington.

LMHC, 1997b, WHC-SD-WM-BIO-001 Revision 0-a, Tank Waste Remediation System Basis for Interim Operation, Lockheed Martin Hanford Corporation, Richland, Washington.

LMHC, 1997c, WHC-SD-WM-JCO-007, Revision 1, Flammable Gas/Slurry Growth Unreviewed Safety Question: Justification For Continued Operation For The Tank Farms at the Hanford Site, Lockheed Martin Hanford Corporation, Richland, Washington.

LMHC, 1997d, WHC-SD-WM-SAD-035, Rev. 0-b, A Safety Assessment of Rotary-Mode Core Sampling In Flammable Gas- Single-Shell Tanks: Hanford Site, Richland, Washington, Lockheed Martin Hanford Corporation, Richland, Washington. 
HNF-SD-WM-SEL-044, Rev. 2

Page 46 of 47

LMHC, 1997e, WHC-SD-WM-OSR-005 Revision O-G, Single Shell Tank Interim Operational Safety Requirements, Lockheed Martin Hanford Corporation, Richland, Washington.

LMHC, 1997f, HNF-SD-WM-TSR-006, Revision 0-B, Tank Waste Remediation System Technical Safety Requirements, Lockheed Martin Hanford Corporation, Richland, Washington.

NFPA, 1996, National Electrical Code, NFPA Standard 70-1996, National Fire Protection Association, Quincy, Massachusetts.

Robinson, 1995, System Design Description for Rotary Mode Core Sampling Exhauster, WHC-SD-WM-SDD-035, Revision 2, Westinghouse Hanford Company, Richland, Washington.

Smalley, 1997, HNF-SD-WM-ETP-230, Revision 2, Engineering Task Plan for the Field Deployment of Two Core Sampling Exhausters Utilizing Basis For Interim Operation Technical Specification Requirements, Fluor Daniel Hanford Company, Richland, Washington.

Wagoner, 1996, DOE RL Letter, J. D. Wagoner to Dr. A.L. Trego, WHC, Authorization of the Safety Assessment of Rotary Mode Core Sampling in Flammable Gas Single Shell Tanks (WHC-SD-WM-SAD-035 Revision 0- $a$ and Interim Operational Safety Requirements, 96QSH-042, August 30, 1996.

Wagoner, 1997a, DOE-RL Letter, J. D. Wagoner to H. J. Hatch, FDH, Contract number DE-AC06-96RL13200; Clarification of Direction Related to the Generic Implications of the Use of Justifications for Continuing Operation (JCOs) in the Unreviewed Safety Question (USQ) Process and its Impact on Push Mode Core Sampling (PMCS), 97-MSD186, March 8, 1997.

Wagoner, 1997b, DOE-RL Letter, J.D. Wagoner to H.J. Hatch, FDH, Contract number DE-AC06-96-RL13200; Approval of Rotary Mode Core Sampling (RMCS) Authorization Basis Change Request 97-WSD-165, July 8, 1997.

Wagoner, 1997c, DOE-RL Letter, J. D. Wagoner to H. J. Hatch, FDH, Contract number DEAC06-96RL13200: Relief From The Commitment to Limit Rotary Mode Core Sampling (RMCS) To Tanks 241-AN-101, 241-AX-103, 241-BX-110, And 241-TY-102, 97-SCD002, dated August 8, 1997.

WHC, 1995, H-2-690001, Rotary Mode Core Sampling Truck Drawing Index, Westinghouse Hanford Company, Richland, Washington. 
HNF-SD-WM-SEL-044, Rev. 2

Page 47 of 47

WHC, 1996b, TWRS Administrative Procedures, WHC-IP-0842, Volume IV, Engineering, Section 5.2, Safety Equipment Lists, Westinghouse Hanford Company, Richland, Washington, July, 1996.

WHC, 1996c, TWRS Administrative Procedures, WHC-IP-0842, Volume IV, Engineering, Section 5.9, Identification of Safety Structures, Systems, and Components, Westinghouse Hanford Company, Richland, Washington, November, 1996. 